\title{
Effect of cellular reservoirs and delays on the global dynamics of HIV
}

\author{
A. M. Elaiw ${ }^{1 *}$, E. K. Elnahary ${ }^{2}$ and A. A. Raezah ${ }^{3}$
}

${ }^{\text {*Correspondence: }}$
a_m_elaiw@yahoo.com
${ }^{1}$ Department of Mathematics,
Faculty of Science, King Abdulaziz
University, Jeddah, Saudi Arabia
Full list of author information is
available at the end of the article

available at the end of the article

\begin{abstract}
We investigate general HIV infection models with three types of infected cells: latently infected cells, long-lived productively infected cells, and short-lived productively infected cells. We incorporate three discrete or distributed time delays into the models. Moreover, we consider the effect of humoral immunity on the dynamical behavior of the HIV. The HIV-target incidence rate, production/proliferation, and removal rates of the cells and HIV are represented by general nonlinear functions. We show that the solutions of the proposed model are nonnegative and ultimately bounded. We derive two threshold parameters which fully determine the existence and stability of the three steady states of the model. Using Lyapunov functionals, we establish the global stability of the steady states of the model. The theoretical results are confirmed by numerical simulations.
\end{abstract}

MSC: 34D20; 34D23; 37N25; 92B05

Keywords: HIV infection; HAART; Global stability; Humoral immune response; Latency; Viral reservoirs 


$$
\begin{aligned}
& \dot{u}(t)=\left(1-\varepsilon_{1}\right) \bar{\lambda}_{3} s(t) p(t)-\beta_{4} u(t), \\
& \dot{p}(t)=\left(1-\varepsilon_{2}\right) \bar{N} \beta_{3} y(t)+\left(1-\varepsilon_{2}\right) \bar{M} \beta_{4} u(t)-\beta_{5} p(t),
\end{aligned}
$$

where $\rho$ is the creation rate of the uninfected $\mathrm{CD} 4^{+} \mathrm{T}$ cells, $\beta_{i}, i=1, \ldots, 5$, are the death rate constants of the five compartments $s, w, y, u$, and $p$, respectively. The model incorporates reverse transcriptase inhibitor (RTI) with efficacy $\varepsilon_{1}$ and protease inhibitor (PI) with efficacy $\varepsilon_{2}$, where $\varepsilon_{1}, \varepsilon_{2} \in[0,1]$. The parameters $\omega$ and $s_{\max }$ are the maximum proliferation rate and the maximum level of concentration of the uninfected $\mathrm{CD} 4^{+} \mathrm{T}$ cells, respectively. The latently infected cells are activated at rate $a_{1} w$. The parameters $\bar{N}$ and $\bar{M}$ are the average numbers of HIV particles generated in the lifetime of the short-lived and long-lived infected cells, respectively. The uninfected $\mathrm{CD} 4^{+} \mathrm{T}$ cells become infected with infectivity $\bar{\lambda}_{1}+\bar{\lambda}_{2}+\bar{\lambda}_{3}$. Elaiw et al. [27] have generalized the above model by incorporating the humoral immune response and considering general nonlinear functions for the generation and removal rates of all compartments:

$$
\begin{aligned}
\dot{s}(t)= & \pi(s(t))-\left(1-\varepsilon_{1}\right)\left(\bar{\lambda}_{1}+\bar{\lambda}_{2}+\bar{\lambda}_{3}\right) \chi(s(t), p(t)), \\
\dot{w}(t)= & \left(1-\varepsilon_{1}\right) \bar{\lambda}_{1} \chi(s(t), p(t))-\left(a_{1}+\beta_{2}\right) g_{1}(w(t)), \\
\dot{y}(t)= & \left(1-\varepsilon_{1}\right) \bar{\lambda}_{2} \chi(s(t), p(t))+a_{1} g_{1}(w(t))-\beta_{3} g_{2}(y(t)), \\
\dot{u}(t)= & \left(1-\varepsilon_{1}\right) \bar{\lambda}_{3} \chi(s(t), p(t))-\beta_{4} g_{3}(u(t)), \\
\dot{p}(t)= & \left(1-\varepsilon_{2}\right) \bar{N} \beta_{3} g_{2}(y(t))+\left(1-\varepsilon_{2}\right) \bar{M} \beta_{4} g_{3}(u(t)) \\
& -\beta_{5} g_{4}(p(t))-q g_{4}(p(t)) g_{5}(x(t)), \\
\dot{x}(t)= & r g_{4}(p(t)) g_{5}(x(t))-\beta_{6} g_{5}(x(t)),
\end{aligned}
$$

where $x$ represents the concentration of the B cells. $\pi, \chi, g_{i}, i=1, \ldots, 5$, are general nonlinear functions. Model (6)-(11) assumes that, once the HIV contacts a CD4 ${ }^{+} \mathrm{T}$ cell, it becomes infected in the same time. Neglecting the time delays is an unrealistic assumption (see, e.g., $[29,30])$.

The aim of this paper is to propose HIV infection models which improve model (6)-(11) by taking into account three time delays, discrete or distributed. We derive two threshold parameters and present some mild sufficient conditions for the existence and global stability of the steady states of the models.

\section{HIV dynamics model with discrete delays}

We formulate a nonlinear HIV dynamics model with latent reservoirs, humoral immunity, and discrete time delays:

$$
\begin{aligned}
& \dot{s}=\pi(s(t))-\left(1-\varepsilon_{1}\right)\left(\bar{\lambda}_{1}+\bar{\lambda}_{2}+\bar{\lambda}_{3}\right) \chi(s(t), p(t)), \\
& \dot{w}=\left(1-\varepsilon_{1}\right) \bar{\lambda}_{1} e^{-\mu_{1} \tau_{1}} \chi\left(s\left(t-\tau_{1}\right), p\left(t-\tau_{1}\right)\right)-\left(a_{1}+\beta_{2}\right) g_{1}(w(t)), \\
& \dot{y}=\left(1-\varepsilon_{1}\right) \bar{\lambda}_{2} e^{-\mu_{2} \tau_{2}} \chi\left(s\left(t-\tau_{2}\right), p\left(t-\tau_{2}\right)\right)+a_{1} g_{1}(w(t))-\beta_{3} g_{2}(y(t)), \\
& \dot{u}=\left(1-\varepsilon_{1}\right) \bar{\lambda}_{3} e^{-\mu_{3} \tau_{3}} \chi\left(s\left(t-\tau_{3}\right), p\left(t-\tau_{3}\right)\right)-\beta_{4} g_{3}(u(t)),
\end{aligned}
$$




$$
\begin{aligned}
& \dot{p}=\left(1-\varepsilon_{2}\right) \bar{N} \beta_{3} g_{2}(y(t))+\left(1-\varepsilon_{2}\right) \bar{M} \beta_{4} g_{3}(u(t))-\beta_{5} g_{4}(p(t))-q g_{4}(p(t)) g_{5}(x(t)), \\
& \dot{x}=r g_{4}(p(t)) g_{5}(x(t))-\beta_{6} g_{5}(x(t)) .
\end{aligned}
$$

All the parameters are positive. Here, $\tau_{1}$ is the time between viral entry and latent infection (i.e., the integration of viral DNA into cell's DNA has finished), while $\tau_{2}$ and $\tau_{3}$ are the times between viral entry and viral production form short-lived productively infected and long-lived productively infected cells, respectively. The factor $e^{-\mu_{i} \tau_{i}}$ accounts for the loss of target cells during the delay period of length $\tau_{i}$, where $\mu_{i}$ is a constant. Let us define $\lambda_{i}=\left(1-\varepsilon_{1}\right) \bar{\lambda}_{i}, i=1,2,3, N=\left(1-\varepsilon_{2}\right) \bar{N}, M=\left(1-\varepsilon_{2}\right) \bar{M}$, and $\lambda=\lambda_{1}+\lambda_{2}+\lambda_{3}$. Functions $\chi$, $\pi, g_{i}, i=1, \ldots, 5$, are continuously differentiable and satisfy the following hypotheses:

(H1). (i) There exists $s_{0}$ such that $\pi\left(s_{0}\right)=0, \pi(s)>0$ for $s \in\left[0, s_{0}\right)$;

(ii) $\pi^{\prime}(s)<0$ for $s \in(0, \infty)$;

(iii) There are $b>0$ and $\bar{b}>0$ such that $\pi(s) \leq b-\bar{b} s$ for $s \in[0, \infty)$.

(H2). (i) $\chi(s, p)>0$ and $\chi(0, p)=\chi(s, 0)=0$ for $s, p \in(0, \infty)$;

(ii) $\frac{\partial \chi(s, p)}{\partial s}>0, \frac{\partial \chi(s, p)}{\partial p}>0$, and $\frac{\partial \chi(s, 0)}{\partial p}>0$ for all $s, p \in(0, \infty)$;

(iii) $\left(\frac{\partial \chi(s, 0)}{\partial p}\right)^{\prime}>0$ for $s \in(0, \infty)$.

(H3). $\quad$ (i) $g_{j}(\eta)>0$ for $\eta \in(0, \infty), g_{j}(0)=0, j=1, \ldots, 5$;

(ii) $g_{j}^{\prime}(\eta)>0$ for $\eta \in(0, \infty), j=1,2,3,5, g_{4}^{\prime}(\eta)>0$, for $\eta \in[0, \infty)$;

(iii) there are $\alpha_{j}>0, j=1, \ldots, 5$, such that $g_{j}(\eta) \geq \alpha_{j} \eta$ for $\eta \in[0, \infty)$.

(H4). (i) $\frac{\partial}{\partial p}\left(\frac{\chi(s, p)}{g_{4}(p)}\right) \leq 0$ for $p \in(0, \infty)$.

We consider system (12)-(17) with the initial conditions:

$$
\begin{aligned}
& s(\theta)=\varphi_{1}(\theta), \quad w(\theta)=\varphi_{2}(\theta), \quad y(\theta)=\varphi_{3}(\theta), \\
& u(\theta)=\varphi_{4}(\theta), \quad p(\theta)=\varphi_{5}(\theta), \quad x(\theta)=\varphi_{6}(\theta), \\
& \varphi_{j}(\theta) \geq 0, \quad \theta \in[-\varsigma, 0], \\
& \varphi_{j}(\theta) \in C\left([-\varsigma, 0], \mathbb{R}_{\geq 0}^{6}\right), \quad j=1, \ldots, 6,
\end{aligned}
$$

where $\varsigma=\max \left\{\tau_{1}, \tau_{2}, \tau_{3}\right\}$ and $C$ is the Banach space of continuous functions mapping the interval $[-\varsigma, 0]$ into $\mathbb{R}_{\geq 0}^{6}$. Then the uniqueness of the solution for $t>0$ is guaranteed [39].

\subsection{Preliminaries}

Lemma 1 Let hypotheses (H1)-(H3) be valid, then the solutions of system (12)-(17) are nonnegative and ultimately bounded.

Proof Let us write system (12)-(17) in the matrix form $\dot{k}(t)=L(k(t))$, where $k=(s, w, y$, $u, p, x)^{T}, L=\left(L_{1}, L_{2}, L_{3}, L_{4}, L_{5}, L_{6}\right)^{T}$, and

$$
L(k(t))=\left(\begin{array}{c}
L_{1}(k(t)) \\
L_{2}(k(t)) \\
L_{3}(k(t)) \\
L_{4}(k(t)) \\
L_{5}(k(t)) \\
L_{6}(k(t))
\end{array}\right),
$$




$$
L=\left(\begin{array}{c}
\pi(s(t))-\lambda \chi(s(t), p(t)) \\
\lambda_{1} e^{-\mu_{1} \tau_{1}} \chi\left(s\left(t-\tau_{1}\right), p\left(t-\tau_{1}\right)\right)-\left(a_{1}+\beta_{2}\right) g_{1}(w(t)) \\
\lambda_{2} e^{-\mu_{2} \tau_{2}} \chi\left(s\left(t-\tau_{2}\right), p\left(t-\tau_{2}\right)\right)+a_{1} g_{1}(w(t))-\beta_{3} g_{2}(y(t)) \\
\lambda_{3} e^{-\mu_{3} \tau_{3}} \chi\left(s\left(t-\tau_{3}\right), p\left(t-\tau_{3}\right)\right)-\beta_{4} g_{3}(u(t)) \\
N \beta_{3} g_{2}(y(t))+M \beta_{4} g_{3}(u(t))-\beta_{5} g_{4}(p(t))-q g_{4}(p(t)) g_{5}(x(t)) \\
r g_{4}(p(t)) g_{5}(x(t))-\beta_{6} g_{5}(x(t))
\end{array}\right) .
$$

We have

$$
\left.L_{i}(k(t))\right|_{k_{i}(t) \in \mathbb{R}_{>0}^{6}} \geq 0, \quad i=1, \ldots, 6 .
$$

Using Lemma 2 in [40], the solutions of system (12)-(17) with the initial states (18) satisfy $X(t) \in \mathbb{R}_{\geq 0}^{6}$ for all $t \geq 0$. The nonnegativity of the model's solution implies that $\lim \sup _{t \rightarrow \infty} s(t) \leq M_{1}$, where $M_{1}=\frac{b}{\bar{b}}$. Let

$$
\begin{aligned}
T(t)= & N e^{-\mu_{1} \tau_{1}} s\left(t-\tau_{1}\right)+N e^{-\mu_{2} \tau_{2}} s\left(t-\tau_{2}\right)+M e^{-\mu_{3} \tau_{3}} s\left(t-\tau_{3}\right) \\
& +N w(t)+N y(t)+M u(t)+\frac{1}{2} p(t)+\frac{q}{2 r} x(t)
\end{aligned}
$$

then

$$
\begin{aligned}
\dot{T}(t)= & N e^{-\mu_{1} \tau_{1}}\left[\pi\left(s\left(t-\tau_{1}\right)\right)-\lambda \chi\left(s\left(t-\tau_{1}\right), p\left(t-\tau_{1}\right)\right)\right] \\
& +N e^{-\mu_{2} \tau_{2}}\left[\pi\left(s\left(t-\tau_{2}\right)\right)-\lambda \chi\left(s\left(t-\tau_{2}\right), p\left(t-\tau_{2}\right)\right)\right] \\
& +M e^{-\mu_{3} \tau_{3}}\left[\pi\left(s\left(t-\tau_{3}\right)\right)-\lambda \chi\left(s\left(t-\tau_{3}\right), p\left(t-\tau_{3}\right)\right)\right] \\
& +N\left[\lambda_{1} e^{-\mu_{1} \tau_{1}} \chi\left(s\left(t-\tau_{1}\right), p\left(t-\tau_{1}\right)\right)-\left(a_{1}+\beta_{2}\right) g_{1}(w(t))\right] \\
& +N\left[\lambda_{2} e^{-\mu_{2} \tau_{2}} \chi\left(s\left(t-\tau_{2}\right), p\left(t-\tau_{2}\right)\right)+a_{1} g_{1}(w(t))-\beta_{3} g_{2}(y(t))\right] \\
& +M\left[\lambda_{3} e^{-\mu_{3} \tau_{3}} \chi\left(s\left(t-\tau_{3}\right), p\left(t-\tau_{3}\right)\right)-\beta_{4} g_{3}(u(t))\right] \\
& +\frac{1}{2}\left(N \beta_{3} g_{2}(y(t))+M \beta_{4} g_{3}(u(t))-\beta_{5} g_{4}(p(t))-q g_{4}(p(t)) g_{5}(x(t))\right) \\
& +\frac{q}{2 r}\left(r g_{4}(p(t)) g_{5}(x(t))-\beta_{6} g_{5}(x(t))\right) \\
\leq & N e^{-\mu_{1} \tau_{1}}\left[b-\bar{b} s\left(t-\tau_{1}\right)\right]+N e^{-\mu_{2} \tau_{2}}\left[b-\overline{b s}\left(t-\tau_{2}\right)\right]+M e^{-\mu_{3} \tau_{3}}\left[b-\bar{b} s\left(t-\tau_{3}\right)\right] \\
& -N \beta_{2} \alpha_{1} w(t)-\frac{1}{2} N \beta_{3} \alpha_{2} y(t)-\frac{1}{2} M \beta_{4} \alpha_{3} u(t)-\frac{1}{2} \beta_{5} \alpha_{4} p(t)-\frac{q}{2 r} \beta_{6} \alpha_{5} p(t) \\
\leq & b\left(N e^{-\mu_{1} \tau_{1}}+N e^{-\mu_{2} \tau_{2}}+M e^{-\mu_{3} \tau_{3}}\right) \\
& -\sigma\left[N e^{-\mu_{1} \tau_{1}} s\left(t-\tau_{1}\right)+N e^{-\mu_{2} \tau_{2}} s\left(t-\tau_{2}\right)+M e^{-\mu_{3} \tau_{3}} s\left(t-\tau_{3}\right)\right. \\
& \left.+N w(t)+N y(t)+M u(t)+\frac{1}{2} p(t)+\frac{q}{2 r} x(t)\right] \\
\leq & b(2 N+M)-\sigma T(t),
\end{aligned}
$$

where $\sigma=\min \left\{\bar{b}, \beta_{2} \alpha_{1}, \frac{1}{2} \beta_{3} \alpha_{2}, \frac{1}{2} \beta_{4} \alpha_{3}, \beta_{5} \alpha_{4}, \beta_{6} \alpha_{5}\right\}$. Then $\limsup _{t \rightarrow \infty} T(t) \leq \frac{b(2 N+M)}{\sigma}$. The nonnegativity of the system's variables implies that

$$
\limsup _{t \rightarrow \infty} w(t) \leq \frac{b(2 N+M)}{N \sigma}=M_{2}
$$




$$
\begin{aligned}
& \limsup _{t \rightarrow \infty} y(t) \leq \frac{b(2 N+M)}{N \sigma}=M_{2}, \\
& \lim \sup _{t \rightarrow \infty} u(t) \leq \frac{b(2 N+M)}{M \sigma}=M_{3}, \\
& \lim \sup _{t \rightarrow \infty} p(t) \leq \frac{2 b(2 N+M)}{\sigma}=M_{4}, \\
& \limsup _{t \rightarrow \infty} x(t) \leq \frac{2 r b(2 N+M)}{q \sigma}=M_{5} .
\end{aligned}
$$

Therefore, $s(t), w(t), y(t), u(t), p(t)$, and $x(t)$ are ultimately bounded.

According to Lemma 1, we can show that the region

$$
\begin{aligned}
\Omega= & \left\{(s, w, y, u, p, x) \in C^{6}:\|s\| \leq M_{1},\|w\| \leq M_{2},\|y\| \leq M_{2},\right. \\
& \left.\|u\| \leq M_{3},\|p\| \leq M_{4},\|x\| \leq M_{5}\right\}
\end{aligned}
$$

is positively invariant with respect to system (12)-(17), where $\|\phi\|=\sup _{t \rightarrow \infty} \phi(t)$.

Lemma 2 Suppose that hypotheses (H1)-(H4) are valid (12)-(17), then there exist two bifurcation parameters $R_{0}$ and $R_{1}$ with $R_{0}>R_{1}>0$ such that

(i) if $R_{0} \leq 1$, then there exists only one steady state $\Pi_{0}$;

(ii) if $R_{1} \leq 1<R_{0}$, then there exist two steady states $\Pi_{0}$ and $\Pi_{1}$;

(iii) if $R_{1}>1$, then there exist three steady states $\Pi_{0}, \Pi_{1}$, and $\Pi_{2}$.

Proof Let $\Pi(s, w, y, u, p, x)$ be any steady state of (12)-(17) satisfying the following equations:

$$
\begin{aligned}
& 0=\pi(s)-\lambda \chi(s, p), \\
& 0=\lambda_{1} e^{-\mu_{1} \tau_{1}} \chi(s, p)-\left(a_{1}+\beta_{2}\right) g_{1}(w), \\
& 0=\lambda_{2} e^{-\mu_{2} \tau_{2}} \chi(s, p)+a_{1} g_{1}(w)-\beta_{3} g_{2}(y), \\
& 0=\lambda_{3} e^{-\mu_{3} \tau_{3}} \chi(s, p)-\beta_{4} g_{3}(u), \\
& 0=N \beta_{3} g_{2}(y)+M \beta_{4} g_{3}(u)-\beta_{5} g_{4}(p)-q g_{4}(p) g_{5}(x), \\
& 0=r g_{4}(p) g_{5}(x)-\beta_{6} g_{5}(x) .
\end{aligned}
$$

From Eq. (25) we have two possible solutions: $g_{5}(x)=0$ and $g_{4}(p)=\beta_{6} / r$. The first possibility $g_{5}(x)=0$ implies that $x=0$. Hypothesis $(\mathrm{H} 3)$ implies that $g_{i}^{-1}, i=1, \ldots, 5$, exist, strictly increasing and $g_{i}^{-1}(0)=0$. Let us define

$$
\begin{aligned}
& \psi_{1}(s)=g_{1}^{-1}\left(\frac{\lambda_{1} e^{-\mu_{1} \tau_{1}}}{\lambda\left(a_{1}+\beta_{2}\right)} \pi(s)\right), \\
& \psi_{2}(s)=g_{2}^{-1}\left(\frac{a_{1} \lambda_{1} e^{-\mu_{1} \tau_{1}}+\left(a_{1}+\beta_{2}\right) \lambda_{2} e^{-\mu_{2} \tau_{2}}}{\beta_{3} \lambda\left(a_{1}+\beta_{2}\right)} \pi(s)\right), \\
& \psi_{3}(s)=g_{3}^{-1}\left(\frac{\lambda_{3} e^{-\mu_{3} \tau_{3}}}{\beta_{4} \lambda} \pi(s)\right), \quad \psi_{4}(s)=g_{4}^{-1}\left(\frac{\gamma}{\lambda} \pi(s)\right),
\end{aligned}
$$


where

$$
\gamma=\frac{N\left(a_{1} \lambda_{1} e^{-\mu_{1} \tau_{1}}+\left(a_{1}+\beta_{2}\right) \lambda_{2} e^{-\mu_{2} \tau_{2}}\right)+M \lambda_{3}\left(a_{1}+\beta_{2}\right) e^{-\mu_{3} \tau_{3}}}{\beta_{5}\left(a_{1}+\beta_{2}\right)} .
$$

It follows from Eqs. (20)-(24) that

$$
w=\psi_{1}(s), \quad y=\psi_{2}(s), \quad u=\psi_{3}(s), \quad p=\psi_{4}(s) .
$$

Obviously, $\psi_{i}(s)>0$ for $s \in\left[0, s_{0}\right)$ and $\psi_{i}\left(s_{0}\right)=0, i=1, \ldots, 4$. From Eqs. (20), (26), and (27) we obtain

$$
\gamma \chi\left(s, \psi_{4}(s)\right)-g_{4}\left(\psi_{4}(s)\right)=0 .
$$

Equation (28) admits a solution $s=s_{0}$ which gives the infection-free steady state $\Pi_{0}\left(s_{0}, 0\right.$, $0,0,0,0)$. Let

$$
\Phi_{1}(s)=\gamma \chi\left(s, \psi_{4}(s)\right)-g_{4}\left(\psi_{4}(s)\right)=0 .
$$

It is clear from hypotheses $(\mathrm{H} 1)$ and $(\mathrm{H} 2)$ that $\Phi_{1}(0)=-g_{4}\left(\psi_{4}(0)\right)<0$ and $\Phi_{1}\left(s_{0}\right)=0$. Moreover,

$$
\Phi_{1}^{\prime}\left(s_{0}\right)=\gamma\left[\frac{\partial \chi\left(s_{0}, 0\right)}{\partial s}+\psi_{4}^{\prime}\left(s_{0}\right) \frac{\partial \chi\left(s_{0}, 0\right)}{\partial p}\right]-g_{4}^{\prime}(0) \psi_{4}^{\prime}\left(s_{0}\right) .
$$

We note from hypothesis $(\mathrm{H} 2)$ that $\frac{\partial \chi\left(s_{0}, 0\right)}{\partial s}=0$. Then

$$
\Phi_{1}^{\prime}\left(s_{0}\right)=\psi_{4}^{\prime}\left(s_{0}\right) g_{4}^{\prime}(0)\left(\frac{\gamma}{g_{4}^{\prime}(0)} \frac{\partial \chi\left(s_{0}, 0\right)}{\partial p}-1\right) .
$$

From Eq. (26), we get

$$
\Phi_{1}^{\prime}\left(s_{0}\right)=\frac{\gamma}{\lambda} \pi^{\prime}\left(s_{0}\right)\left(\frac{\gamma}{g_{4}^{\prime}(0)} \frac{\partial \chi\left(s_{0}, 0\right)}{\partial p}-1\right) .
$$

Hence, from hypothesis (H1), we have $\pi^{\prime}\left(s_{0}\right)<0$. Therefore, if $\frac{\gamma}{g_{4}^{\prime}(0)} \frac{\partial \chi\left(s_{0}, 0\right)}{\partial p}>1$, then $\Phi_{1}^{\prime}\left(s_{0}\right)<$ 0 , and there exists $s_{1} \in\left(0, s_{0}\right)$ such that $\Phi_{1}\left(s_{1}\right)=0$. Hypotheses $(\mathrm{H} 1)-(\mathrm{H} 3)$ imply that

$$
w_{1}=\psi_{1}\left(s_{1}\right)>0, \quad y_{1}=\psi_{2}\left(s_{1}\right)>0, \quad u_{1}=\psi_{3}\left(s_{1}\right)>0, \quad p_{1}=\psi_{4}\left(s_{1}\right)>0 .
$$

It means that a humoral-inactivated infection steady state $\Pi_{1}\left(s_{1}, w_{1}, y_{1}, u_{1}, p_{1}, 0\right)$ exists when $\frac{\gamma}{g_{4}^{\prime}(0)} \frac{\partial \chi\left(s_{0}, 0\right)}{\partial p}>1$. Let us define the basic infection reproduction number as follows:

$$
R_{0}=\frac{\gamma}{g_{4}^{\prime}(0)} \frac{\partial \chi\left(s_{0}, 0\right)}{\partial p}
$$

The other solution of Eq. (25) is $g_{4}\left(p_{2}\right)=\frac{\beta_{6}}{r}$, which yields $p_{2}=g_{4}^{-1}\left(\frac{\beta_{6}}{r}\right)>0$. Substitute $p=p_{2}$ in Eq. (20) and let $\Phi_{2}(s)=\pi(s)-\lambda \chi\left(s, p_{2}\right)=0$. According to hypotheses (H1) and (H2), 
$\Phi_{2}$ is strictly decreasing, $\Phi_{2}(0)=\pi(0)>0$ and $\Phi_{2}\left(s_{0}\right)=-\lambda \chi\left(s_{0}, p_{2}\right)<0$. Thus, there exists unique $s_{2} \in\left(0, s_{0}\right)$ such that $\Phi_{2}\left(s_{2}\right)=0$. It follows from Eqs. (24) and (27) that

$$
\begin{aligned}
& w_{2}=\psi_{1}\left(s_{2}\right)>0, \quad y_{2}=\psi_{2}\left(s_{2}\right)>0, \quad u_{2}=\psi_{3}\left(s_{2}\right)>0, \quad p_{2}=g_{4}^{-1}\left(\frac{\beta_{6}}{r}\right)>0, \\
& x_{2}=g_{5}^{-1}\left(\frac{\beta_{5}}{q}\left(\gamma \frac{\chi\left(s_{2}, p_{2}\right)}{g_{4}\left(p_{2}\right)}-1\right)\right) .
\end{aligned}
$$

Thus, $x_{2}>0$ when $\gamma \frac{\chi\left(s_{2}, p_{2}\right)}{g_{4}\left(p_{2}\right)}>1$. Now we define the humoral immune response activation number as follows:

$$
R_{1}=\gamma \frac{\chi\left(s_{2}, p_{2}\right)}{g_{4}\left(p_{2}\right)}
$$

If $R_{1}>1$, then $x_{2}=g_{5}^{-1}\left(\frac{\beta_{5}}{q}\left(R_{1}-1\right)\right)>0$, and there exists a humoral-activated infection steady state $\Pi_{2}\left(s_{2}, w_{2}, y_{2}, u_{2}, p_{2}, x_{2}\right)$.

Clearly, from hypotheses (H2) and (H4), we have

$$
R_{1}=\gamma \frac{\chi\left(s_{2}, p_{2}\right)}{g_{4}\left(p_{2}\right)} \leq \gamma \lim _{p \rightarrow 0^{+}} \frac{\chi\left(s_{2}, p\right)}{g_{4}(p)}=\frac{\gamma}{g_{4}^{\prime}(0)} \frac{\partial \chi\left(s_{2}, 0\right)}{\partial p}<\frac{\gamma}{g_{4}^{\prime}(0)} \frac{\partial \chi\left(s_{0}, 0\right)}{\partial p}=R_{0} .
$$

We will use the following equalities throughout the paper:

$$
\begin{aligned}
& \ln (\left.\frac{\chi\left(s\left(t-\tau_{1}\right), p\left(t-\tau_{1}\right)\right)}{\chi(s, p)}\right) \\
&= \ln \left(\frac{g_{1}(\hat{w}) \chi\left(s\left(t-\tau_{1}\right), p\left(t-\tau_{1}\right)\right)}{g_{1}(w) \chi(\hat{s}, \hat{p})}\right)+\ln \left(\frac{\chi(\hat{s}, \hat{p})}{\chi(s, \hat{p})}\right) \\
& \quad+\ln \left(\frac{g_{4}(p) \chi(s, \hat{p})}{g_{4}(\hat{p}) \chi(s, p)}\right)+\ln \left(\frac{g_{4}(\hat{p}) g_{2}(y)}{g_{4}(p) g_{2}(\hat{y})}\right)+\ln \left(\frac{g_{2}(\hat{y}) g_{1}(w)}{g_{2}(y) g_{1}(\hat{w})}\right), \\
& \ln \left(\frac{\chi\left(s\left(t-\tau_{2}\right), p\left(t-\tau_{2}\right)\right)}{\chi(s, p)}\right) \\
&=\ln \left(\frac{g_{2}(\hat{y}) \chi\left(s\left(t-\tau_{2}\right), p\left(t-\tau_{2}\right)\right)}{g_{2}(y) \chi(\hat{s}, \hat{p})}\right)+\ln \left(\frac{\chi(\hat{s}, \hat{p})}{\chi(s, \hat{p})}\right) \\
& \quad+\ln \left(\frac{g_{4}(\hat{p}) g_{2}(y)}{g_{4}(p) g_{2}(\hat{y})}\right)+\ln \left(\frac{g_{4}(p) \chi(s, \hat{p})}{g_{4}(\hat{p}) \chi(s, p)}\right) \\
& \ln \left(\frac{\chi\left(s\left(t-\tau_{3}\right), p\left(t-\tau_{3}\right)\right)}{\chi(s, p)}\right) \\
&=\ln \left(\frac{g_{3}(\hat{u}) \chi\left(s\left(t-\tau_{3}\right), p\left(t-\tau_{3}\right)\right)}{g_{3}(u) \chi(\hat{s}, \hat{p})}\right)+\ln \left(\frac{\chi(\hat{s}, \hat{p})}{\chi(s, \hat{p})}\right) \\
& \quad+\ln \left(\frac{g_{3}(u) g_{4}(\hat{p})}{g_{3}(\hat{u}) g_{4}(p)}\right)+\ln \left(\frac{g_{4}(p) \chi(s, \hat{p})}{g_{4}(\hat{p}) \chi(s, p)}\right) .
\end{aligned}
$$

\subsection{Global properties}

The following theorems investigate the global stability of the steady states of system (12)(17).

Let us define the function $F:(0, \infty) \rightarrow[0, \infty)$ as $F(z)=z-1-\ln z$. Denote $(s, w, y, u$, $p, x)=(s(t), w(t), y(t), u(t), p(t), x(t))$. 
Theorem 1 If $R_{0} \leq 1$ and hypotheses $(\mathrm{H} 1)-(\mathrm{H} 4)$ are valid, then $\Pi_{0}$ is globally asymptotically stable (GAS).

Proof Define a Lyapunov functional $V_{0}$ as follows:

$$
\begin{aligned}
V_{0}= & s-s_{0}-\int_{s_{0}}^{s} \lim _{p \rightarrow 0^{+}} \frac{\chi\left(s_{0}, p\right)}{\chi(\eta, p)} d \eta+\ell_{1} w+\ell_{2} y+\ell_{3} u \\
& +\ell_{1} \int_{0}^{\tau_{1}} \lambda_{1} e^{-\mu_{1} \tau_{1}} \chi(s(t-\theta), p(t-\theta)) d \theta \\
& +\ell_{2} \int_{0}^{\tau_{2}} \lambda_{2} e^{-\mu_{2} \tau_{2}} \chi(s(t-\theta), p(t-\theta)) d \theta \\
& +\ell_{3} \int_{0}^{\tau_{3}} \lambda_{3} e^{-\mu_{3} \tau_{3}} \chi(s(t-\theta), p(t-\theta)) d \theta+\ell_{4} p+\ell_{5} x,
\end{aligned}
$$

where

$$
\begin{aligned}
& \lambda=\lambda_{1} \ell_{1} e^{-\mu_{1} \tau_{1}}+\lambda_{2} \ell_{2} e^{-\mu_{2} \tau_{2}}+\lambda_{3} \ell_{3} e^{-\mu_{3} \tau_{3}}, \quad\left(a_{1}+\beta_{2}\right) \ell_{1}=a_{1} \ell_{2}, \\
& \ell_{2}=N \ell_{4}, \quad \ell_{3}=M \ell_{4}, \quad q \ell_{4}=r \ell_{5} .
\end{aligned}
$$

The solution of Eqs. (31) is given by

$$
\ell_{1}=\frac{a_{1} N \lambda}{\gamma \beta_{5}\left(a_{1}+\beta_{2}\right)}, \quad \ell_{2}=\frac{N \lambda}{\gamma \beta_{5}}, \quad \ell_{3}=\frac{M \lambda}{\gamma \beta_{5}}, \quad \ell_{4}=\frac{\lambda}{\gamma \beta_{5}}, \quad \ell_{5}=\frac{q \lambda}{r \gamma \beta_{5}} .
$$

We calculate $\frac{d V_{0}}{d t}$ along the trajectories of (12)-(17) as follows:

$$
\begin{aligned}
\frac{d V_{0}}{d t}= & \left(1-\lim _{p \rightarrow 0^{+}} \frac{\chi\left(s_{0}, p\right)}{\chi(s, p)}\right)(\pi(s)-\lambda \chi(s, p)) \\
& +\ell_{1}\left(\lambda_{1} e^{-\mu_{1} \tau_{1}} \chi\left(s\left(t-\tau_{1}\right), p\left(t-\tau_{1}\right)\right)-\left(a_{1}+\beta_{2}\right) g_{1}(w)\right) \\
& +\ell_{2}\left(\lambda_{2} e^{-\mu_{2} \tau_{2}} \chi\left(s\left(t-\tau_{2}\right), p\left(t-\tau_{2}\right)\right)+a_{1} g_{1}(w)-\beta_{3} g_{2}(y)\right) \\
& +\ell_{3}\left(\lambda_{3} e^{-\mu_{3} \tau_{3}} \chi\left(s\left(t-\tau_{3}\right), p\left(t-\tau_{3}\right)\right)-\beta_{4} g_{3}(u)\right) \\
& +\ell_{1} \lambda_{1} e^{-\mu_{1} \tau_{1}}\left(\chi(s, p)-\chi\left(s\left(t-\tau_{1}\right), p\left(t-\tau_{1}\right)\right)\right) \\
& +\ell_{2} \lambda_{2} e^{-\mu_{2} \tau_{2}}\left(\chi(s, p)-\chi\left(s\left(t-\tau_{2}\right), p\left(t-\tau_{2}\right)\right)\right) \\
& +\ell_{3} \lambda_{3} e^{-\mu_{3} \tau_{3}}\left(\chi(s, p)-\chi\left(s\left(t-\tau_{3}\right), p\left(t-\tau_{3}\right)\right)\right) \\
& +\ell_{4}\left(N \beta_{3} g_{2}(y)+M \beta_{4} g_{3}(u)-\beta_{5} g_{4}(p)-q g_{4}(p) g_{5}(x)\right) \\
& +\ell_{5}\left(r g_{4}(p) g_{5}(x)-\beta_{6} g_{5}(x)\right) .
\end{aligned}
$$

Collecting terms of Eq. (33) and using $\pi\left(s_{0}\right)=0$, we obtain

$$
\begin{aligned}
\frac{d V_{0}}{d t}= & \left(\pi(s)-\pi\left(s_{0}\right)\right)\left(1-\lim _{p \rightarrow 0^{+}} \frac{\chi\left(s_{0}, p\right)}{\chi(s, p)}\right) \\
& +\left(\frac{\lambda \chi(s, p)}{g_{4}(p)} \lim _{p \rightarrow 0^{+}} \frac{\chi\left(s_{0}, p\right)}{\chi(s, p)}-\ell_{4} \beta_{5}\right) g_{4}(p)-\ell_{5} \beta_{6} g_{5}(x) \\
\leq & \left(\pi(s)-\pi\left(s_{0}\right)\right)\left(1-\lim _{p \rightarrow 0^{+}} \frac{\chi\left(s_{0}, p\right)}{\chi(s, p)}\right)
\end{aligned}
$$




$$
\begin{aligned}
& +\left(\lim _{p \rightarrow 0^{+}} \frac{\lambda \chi(s, p)}{g_{4}(p)} \lim _{p \rightarrow 0^{+}} \frac{\chi\left(s_{0}, p\right)}{\chi(s, p)}-\ell_{4} \beta_{5}\right) g_{4}(p)-\ell_{5} \beta_{6} g_{5}(x) \\
= & \left(\pi(s)-\pi\left(s_{0}\right)\right)\left(1-\frac{\partial \chi\left(s_{0}, 0\right) / \partial p}{\partial \chi(s, 0) / \partial p}\right) \\
& +\ell_{4} \beta_{5}\left(\frac{\lambda}{\ell_{4} \beta_{5} g_{4}^{\prime}(0)} \frac{\partial \chi\left(s_{0}, 0\right)}{\partial p}-1\right) g_{4}(p)-\ell_{5} \beta_{6} g_{5}(x) \\
= & \left(\pi(s)-\pi\left(s_{0}\right)\right)\left(1-\frac{\partial \chi\left(s_{0}, 0\right) / \partial p}{\partial \chi(s, 0) / \partial p}\right)+\ell_{4} \beta_{5}\left(R_{0}-1\right) g_{4}(p)-\ell_{5} \beta_{6} g_{5}(x) .
\end{aligned}
$$

By hypotheses (H1) and (H2), we obtain

$$
\left(\pi(s)-\pi\left(s_{0}\right)\right)\left(1-\frac{\partial \chi\left(s_{0}, 0\right) / \partial p}{\partial \chi(s, 0) / \partial p}\right) \leq 0 .
$$

Therefore, if $R_{0} \leq 1$, then $\frac{d V_{0}}{d t} \leq 0$ for $s, p, x>0$. Clearly, $\frac{d V_{0}}{d t}=0$ at $\Pi_{0}$. Applying LaSalle's invariance principle (LIP), we get that $\Pi_{0}$ is GAS.

Lemma 3 If $R_{0}>1$ and hypotheses $(\mathrm{H} 1)-(\mathrm{H} 4)$ are valid, then

$$
\operatorname{sgn}\left(R_{1}-1\right)=\operatorname{sgn}\left(p_{1}-p_{2}\right)=\operatorname{sgn}\left(s_{2}-s_{1}\right) .
$$

Proof Using hypotheses (H1) and (H2), for $s_{1}, s_{2}, p_{1}, p_{2}>0$, we get

$$
\begin{aligned}
& \left(s_{1}-s_{2}\right)\left(\pi\left(s_{2}\right)-\pi\left(s_{1}\right)\right)>0, \\
& \left(s_{2}-s_{1}\right)\left(\chi\left(s_{2}, p_{2}\right)-\chi\left(s_{1}, p_{2}\right)\right)>0, \\
& \left(p_{2}-p_{1}\right)\left(\chi\left(s_{1}, p_{2}\right)-\chi\left(s_{1}, p_{1}\right)\right)>0,
\end{aligned}
$$

and from hypothesis (H4), we obtain

$$
\left(p_{1}-p_{2}\right)\left(\frac{\chi\left(s_{1}, p_{2}\right)}{g_{2}\left(p_{2}\right)}-\frac{\chi\left(s_{1}, p_{1}\right)}{g_{2}\left(p_{1}\right)}\right)>0
$$

First, we show that $\operatorname{sgn}\left(p_{1}-p_{2}\right)=\operatorname{sgn}\left(s_{2}-s_{1}\right)$. Suppose that $\operatorname{sgn}\left(p_{2}-p_{1}\right)=\operatorname{sgn}\left(s_{2}-s_{1}\right)$. Using the steady state conditions of $\Pi_{1}$ and $\Pi_{2}$, we obtain

$$
\begin{aligned}
\pi\left(s_{2}\right)-\pi\left(s_{1}\right) & =\lambda\left[\chi\left(s_{2}, p_{2}\right)-\chi\left(s_{1}, p_{1}\right)\right] \\
& =\lambda\left[\left(\chi\left(s_{2}, p_{2}\right)-\chi\left(s_{1}, p_{2}\right)\right)+\left(\chi\left(s_{1}, p_{2}\right)-\chi\left(s_{1}, p_{1}\right)\right)\right] .
\end{aligned}
$$

Therefore, from inequalities (34)-(36) we obtain $\operatorname{sgn}\left(s_{2}-s_{1}\right)=\operatorname{sgn}\left(s_{1}-s_{2}\right)$, which is a contradiction; hence, $\operatorname{sgn}\left(p_{1}-p_{2}\right)=\operatorname{sgn}\left(s_{2}-s_{1}\right)$. Using Eq. (29) and the definition of $R_{1}$, we get

$$
\begin{aligned}
R_{1}-1 & =\gamma\left(\frac{\chi\left(s_{2}, p_{2}\right)}{g_{4}\left(p_{2}\right)}-\frac{\chi\left(s_{1}, p_{1}\right)}{g_{4}\left(p_{1}\right)}\right) \\
& =\gamma\left[\frac{1}{g_{4}\left(p_{2}\right)}\left(\chi\left(s_{2}, p_{2}\right)-\chi\left(s_{1}, p_{2}\right)\right)+\frac{\chi\left(s_{1}, p_{2}\right)}{g_{4}\left(p_{2}\right)}-\frac{\chi\left(s_{1}, p_{1}\right)}{g_{4}\left(p_{1}\right)}\right] .
\end{aligned}
$$

Thus, from Eqs. (35) and (37) we obtain $\operatorname{sgn}\left(R_{1}-1\right)=\operatorname{sgn}\left(p_{1}-p_{2}\right)$. 
Theorem 2 If $R_{1} \leq 1<R_{0}$ and hypotheses $(\mathrm{H} 1)-(\mathrm{H} 4)$ are valid, then $\Pi_{1}$ is GAS.

Proof Let

$$
\begin{aligned}
V_{1}= & -s_{1}-\int_{s_{1}}^{s} \frac{\chi\left(s_{1}, p_{1}\right)}{\chi\left(\eta, p_{1}\right)} d \eta+\ell_{1}\left(w-w_{1}-\int_{w_{1}}^{w} \frac{g_{1}\left(w_{1}\right)}{g_{1}(\eta)} d \eta\right) \\
& +\ell_{2}\left(y-y_{1}-\int_{y_{1}}^{y} \frac{g_{2}\left(y_{1}\right)}{g_{2}(\eta)} d \eta\right)+\ell_{3}\left(u-u_{1}-\int_{u_{1}}^{u} \frac{g_{3}\left(u_{1}\right)}{g_{3}(\eta)} d \eta\right) \\
& +\ell_{1} \lambda_{1} \chi\left(s_{1}, p_{1}\right) e^{-\mu_{1} \tau_{1}} \int_{0}^{\tau_{1}} F\left(\frac{\chi(s(t-\theta), p(t-\theta))}{\chi\left(s_{1}, p_{1}\right)}\right) d \theta \\
& +\ell_{2} \lambda_{2} \chi\left(s_{1}, p_{1}\right) e^{-\mu_{2} \tau_{2}} \int_{0}^{\tau_{2}} F\left(\frac{\chi(s(t-\theta), p(t-\theta))}{\chi\left(s_{1}, p_{1}\right)}\right) d \theta \\
& +\ell_{3} \lambda_{3} \chi\left(s_{1}, p_{1}\right) e^{-\mu_{3} \tau_{3}} \int_{0}^{\tau_{3}} F\left(\frac{\chi(s(t-\theta), p(t-\theta))}{\chi\left(s_{1}, p_{1}\right)}\right) d \theta \\
& +\ell_{4}\left(p-p_{1}-\int_{p_{1}}^{p} \frac{g_{4}\left(p_{1}\right)}{g_{4}(\eta)} d \eta\right)+\ell_{5} x .
\end{aligned}
$$

Calculating $\frac{d V_{1}}{d t}$ along the solutions of (12)-(17), we obtain

$$
\begin{aligned}
& \frac{d V_{1}}{d t}=\left(1-\frac{\chi\left(s_{1}, p_{1}\right)}{\chi\left(s, p_{1}\right)}\right)(\pi(s)-\lambda \chi(s, p)) \\
& +\ell_{1}\left(1-\frac{g_{1}\left(w_{1}\right)}{g_{1}(w)}\right)\left(\lambda_{1} e^{-\mu_{1} \tau_{1}} \chi\left(s\left(t-\tau_{1}\right), p\left(t-\tau_{1}\right)\right)-\left(a_{1}+\beta_{2}\right) g_{1}(w)\right) \\
& +\ell_{2}\left(1-\frac{g_{2}\left(y_{1}\right)}{g_{2}(y)}\right)\left(\lambda_{2} e^{-\mu_{2} \tau_{2}} \chi\left(s\left(t-\tau_{2}\right), p\left(t-\tau_{2}\right)\right)+a_{1} g_{1}(w)-\beta_{3} g_{2}(y)\right) \\
& +\ell_{3}\left(1-\frac{g_{3}\left(u_{1}\right)}{g_{3}(u)}\right)\left(\lambda_{3} e^{-\mu_{3} \tau_{3}} \chi\left(s\left(t-\tau_{3}\right), p\left(t-\tau_{3}\right)\right)-\beta_{4} g_{3}(u)\right) \\
& +\ell_{1} \lambda_{1} e^{-\mu_{1} \tau_{1}}\left(\chi(s, p)-\chi\left(s\left(t-\tau_{1}\right), p\left(t-\tau_{1}\right)\right)\right) \\
& +\ell_{1} \lambda_{1} \chi\left(s_{1}, p_{1}\right) e^{-\mu_{1} \tau_{1}} \ln \left(\frac{\chi\left(s\left(t-\tau_{1}\right), p\left(t-\tau_{1}\right)\right)}{\chi(s, p)}\right) \\
& +\ell_{2} \lambda_{2} e^{-\mu_{2} \tau_{2}}\left(\chi(s, p)-\chi\left(s\left(t-\tau_{2}\right), p\left(t-\tau_{2}\right)\right)\right) \\
& +\ell_{2} \lambda_{2} \chi\left(s_{1}, p_{1}\right) e^{-\mu_{2} \tau_{2}} \ln \left(\frac{\chi\left(s\left(t-\tau_{2}\right), p\left(t-\tau_{2}\right)\right)}{\chi(s, p)}\right) \\
& +\ell_{3} \lambda_{3} e^{-\mu_{3} \tau_{3}}\left(\chi(s, p)-\chi\left(s\left(t-\tau_{3}\right), p\left(t-\tau_{3}\right)\right)\right) \\
& +\ell_{3} \lambda_{3} \chi\left(s_{1}, p_{1}\right) e^{-\mu_{3} \tau_{3}} \ln \left(\frac{\chi\left(s\left(t-\tau_{3}\right), p\left(t-\tau_{3}\right)\right)}{\chi(s, p)}\right) \\
& +\ell_{4}\left(1-\frac{g_{4}\left(p_{1}\right)}{g_{4}(p)}\right)\left(N \beta_{3} g_{2}(y)+M \beta_{4} g_{3}(u)-\beta_{5} g_{4}(p)-q g_{4}(p) g_{5}(x)\right) \\
& +\ell_{5}\left(r g_{4}(p) g_{5}(x)-\beta_{6} g_{5}(x)\right) \text {. }
\end{aligned}
$$

Collecting terms of Eq. (38) and applying the conditions of the steady state $\Pi_{1}$

$$
\pi\left(s_{1}\right)=\lambda \chi\left(s_{1}, p_{1}\right)
$$




$$
\begin{aligned}
& \left(a_{1}+\beta_{2}\right) g_{1}\left(w_{1}\right)=\lambda_{1} e^{-\mu_{1} \tau_{1}} \chi\left(s_{1}, p_{1}\right) \\
& \ell_{2} \beta_{3} g_{2}\left(y_{1}\right)=\left(\ell_{1} \lambda_{1} e^{-\mu_{1} \tau_{1}}+\ell_{2} \lambda_{2} e^{-\mu_{2} \tau_{2}}\right) \chi\left(s_{1}, p_{1}\right) \\
& \beta_{4} g_{3}\left(u_{1}\right)=\lambda_{3} e^{-\mu_{3} \tau_{3}} \chi\left(s_{1}, p_{1}\right), \quad \ell_{4} \beta_{5} g_{4}\left(p_{1}\right)=\lambda \chi\left(s_{1}, p_{1}\right)
\end{aligned}
$$

we get

$$
\begin{aligned}
& \frac{d V_{1}}{d t}=\left(\pi(s)-\pi\left(s_{1}\right)\right)\left(1-\frac{\chi\left(s_{1}, p_{1}\right)}{\chi\left(s, p_{1}\right)}\right)+\lambda \chi\left(s_{1}, p_{1}\right)\left(1-\frac{\chi\left(s_{1}, p_{1}\right)}{\chi\left(s, p_{1}\right)}\right) \\
& +\lambda \chi\left(s_{1}, p_{1}\right)\left(\frac{\chi(s, p)}{\chi\left(s, p_{1}\right)}-\frac{g_{4}(p)}{g_{4}\left(p_{1}\right)}\right) \\
& -\ell_{1} \lambda_{1} e^{-\mu_{1} \tau_{1}} \chi\left(s_{1}, p_{1}\right) \frac{g_{1}\left(w_{1}\right) \chi\left(s\left(t-\tau_{1}\right), p\left(t-\tau_{1}\right)\right)}{g_{1}(w) \chi\left(s_{1}, p_{1}\right)}+\ell_{1} \lambda_{1} e^{-\mu_{1} \tau_{1}} \chi\left(s_{1}, p_{1}\right) \\
& -\ell_{2} \lambda_{2} e^{-\mu_{2} \tau_{2}} \chi\left(s_{1}, p_{1}\right) \frac{g_{2}\left(y_{1}\right) \chi\left(s\left(t-\tau_{2}\right), p\left(t-\tau_{2}\right)\right)}{g_{2}(y) \chi\left(s_{1}, p_{1}\right)} \\
& -\ell_{1} \lambda_{1} e^{-\mu_{1} \tau_{1}} \chi\left(s_{1}, p_{1}\right) \frac{g_{2}\left(y_{1}\right) g_{1}(w)}{g_{2}(y) g_{1}\left(w_{1}\right)} \\
& +\left(\ell_{1} \lambda_{1} e^{-\mu_{1} \tau_{1}}+\ell_{2} \lambda_{2} e^{-\mu_{2} \tau_{2}}\right) \chi\left(s_{1}, p_{1}\right) \\
& -\ell_{3} \lambda_{3} e^{-\mu_{3} \tau_{3}} \chi\left(s_{1}, p_{1}\right) \frac{g_{3}\left(u_{1}\right) \chi\left(s\left(t-\tau_{3}\right), p\left(t-\tau_{3}\right)\right)}{\chi\left(s_{1}, p_{1}\right) g_{3}(u)} \\
& +\ell_{3} \lambda_{3} e^{-\mu_{3} \tau_{3}} \chi\left(s_{1}, p_{1}\right)+\ell_{1} \lambda_{1} e^{-\mu_{1} \tau_{1}} \chi\left(s_{1}, p_{1}\right) \ln \left(\frac{\chi\left(s\left(t-\tau_{1}\right), p\left(t-\tau_{1}\right)\right)}{\chi(s, p)}\right) \\
& +\ell_{2} \lambda_{2} e^{-\mu_{2} \tau_{2}} \chi\left(s_{1}, p_{1}\right) \ln \left(\frac{\chi\left(s\left(t-\tau_{2}\right), p\left(t-\tau_{2}\right)\right)}{\chi(s, p)}\right) \\
& +\ell_{3} \lambda_{3} e^{-\mu_{3} \tau_{3}} \chi\left(s_{1}, p_{1}\right) \ln \left(\frac{\chi\left(s\left(t-\tau_{3}\right), p\left(t-\tau_{3}\right)\right)}{\chi(s, p)}\right) \\
& -\left(\ell_{1} \lambda_{1} e^{-\mu_{1} \tau_{1}}+\ell_{2} \lambda_{2} e^{-\mu_{2} \tau_{2}}\right) \chi\left(s_{1}, p_{1}\right) \frac{g_{2}(y) g_{4}\left(p_{1}\right)}{g_{2}\left(y_{1}\right) g_{4}(p)} \\
& -\ell_{3} \lambda_{3} e^{-\mu_{3} \tau_{3}} \chi\left(s_{1}, p_{1}\right) \frac{g_{3}(u) g_{4}\left(p_{1}\right)}{g_{3}\left(u_{1}\right) g_{4}(p)} \\
& +\lambda \chi\left(s_{1}, p_{1}\right)+r \ell_{5}\left(g_{4}\left(p_{1}\right)-\frac{\beta_{6}}{r}\right) g_{5}(x) \text {. }
\end{aligned}
$$

Using inequalities (30) with $\hat{s}=s_{1}, \hat{w}=w_{1}, \hat{y}=y_{1}$, and $\hat{p}=p_{1}$, we can obtain

$$
\begin{aligned}
\frac{d V_{1}}{d t}= & \left(\pi(s)-\pi\left(s_{1}\right)\right)\left(1-\frac{\chi\left(s_{1}, p_{1}\right)}{\chi\left(s, p_{1}\right)}\right)+\lambda \chi\left(s_{1}, p_{1}\right)\left(\frac{\chi(s, p)}{\chi\left(s, p_{1}\right)}-\frac{g_{4}(p)}{g_{4}\left(p_{1}\right)}\right)\left(1-\frac{\chi\left(s, p_{1}\right)}{\chi(s, p)}\right) \\
& -\lambda \chi\left(s_{1}, p_{1}\right)\left[F\left(\frac{\chi\left(s_{1}, p_{1}\right)}{\chi\left(s, p_{1}\right)}\right)+F\left(\frac{g_{4}(p) \chi\left(s, p_{1}\right)}{g_{4}\left(p_{1}\right) \chi(s, p)}\right)\right] \\
& -\ell_{1} \lambda_{1} e^{-\mu_{1} \tau_{1}} \chi\left(s_{1}, p_{1}\right)\left[F\left(\frac{g_{1}\left(w_{1}\right) \chi\left(s\left(t-\tau_{1}\right), p\left(t-\tau_{1}\right)\right)}{g_{1}(w) \chi\left(s_{1}, p_{1}\right)}\right)+F\left(\frac{g_{2}\left(y_{1}\right) g_{1}(w)}{g_{2}(y) g_{1}\left(w_{1}\right)}\right)\right] \\
& -\ell_{2} \lambda_{2} e^{-\mu_{2} \tau_{2}} \chi\left(s_{1}, p_{1}\right) F\left(\frac{g_{2}\left(y_{1}\right) \chi\left(s\left(t-\tau_{2}\right), p\left(t-\tau_{2}\right)\right)}{g_{2}(y) \chi\left(s_{1}, p_{1}\right)}\right) \\
& -\ell_{3} \lambda_{3} e^{-\mu_{3} \tau_{3}} \chi\left(s_{1}, p_{1}\right)\left[F\left(\frac{g_{3}\left(u_{1}\right) \chi\left(s\left(t-\tau_{3}\right), p\left(t-\tau_{3}\right)\right)}{g_{3}(u) \chi\left(s_{1}, p_{1}\right)}\right)+F\left(\frac{g_{3}(u) g_{4}\left(p_{1}\right)}{g_{3}\left(u_{1}\right) g_{4}(p)}\right)\right]
\end{aligned}
$$




$$
\begin{aligned}
& -\left(\ell_{1} \lambda_{1} e^{-\mu_{1} \tau_{1}}+\ell_{2} \lambda_{2} e^{-\mu_{2} \tau_{2}}\right) \chi\left(s_{1}, p_{1}\right) F\left(\frac{g_{2}(y) g_{4}\left(p_{1}\right)}{g_{2}\left(y_{1}\right) g_{4}(p)}\right) \\
& +r \ell_{5}\left(g_{4}\left(p_{1}\right)-g_{4}\left(p_{2}\right)\right) g_{5}(x) .
\end{aligned}
$$

Hypotheses (H1), (H2), (H4), Lemma 3, and the condition $R_{1} \leq 1$ imply that

$$
\begin{aligned}
& \left(\pi(s)-\pi\left(s_{1}\right)\right)\left(1-\frac{\chi\left(s_{1}, p_{1}\right)}{\chi\left(s, p_{1}\right)}\right) \leq 0, \\
& \left(\frac{\chi(s, p)}{\chi\left(s, p_{1}\right)}-\frac{g_{4}(p)}{g_{4}\left(p_{1}\right)}\right)\left(1-\frac{\chi\left(s, p_{1}\right)}{\chi(s, p)}\right) \leq 0, \\
& g_{4}\left(p_{1}\right)-g_{4}\left(p_{2}\right) \leq 0 .
\end{aligned}
$$

It follows that, for all $s, y, p, x>0$, we have $\frac{d V_{1}}{d t} \leq 0$ and $\frac{d V_{1}}{d t}=0$ at $\Pi_{1}$. By LIP $\Pi_{1}$ is GAS.

Theorem 3 If $R_{1}>1$ and hypotheses $(\mathrm{H} 1)-(\mathrm{H} 4)$ are valid, then $\Pi_{2}$ is GAS.

Proof Define

$$
\begin{aligned}
V_{2}= & s-s_{2}-\int_{s_{2}}^{s} \frac{\chi\left(s_{2}, p_{2}\right)}{\chi\left(\eta, p_{2}\right)} d \eta+\ell_{1}\left(w-w_{2}-\int_{w_{2}}^{w} \frac{g_{1}\left(w_{2}\right)}{g_{1}(\eta)} d \eta\right) \\
& +\ell_{2}\left(y-y_{2}-\int_{y_{2}}^{y} \frac{g_{2}\left(y_{2}\right)}{g_{2}(\eta)} d \eta\right)+\ell_{3}\left(u-u_{2}-\int_{u_{2}}^{u} \frac{g_{3}\left(u_{2}\right)}{g_{3}(\eta)} d \eta\right) \\
& +\ell_{1} \lambda_{1} \chi\left(s_{2}, p_{2}\right) e^{-\mu_{1} \tau_{1}} \int_{0}^{\tau_{1}} F\left(\frac{\chi(s(t-\theta), p(t-\theta))}{\chi\left(s_{2}, p_{2}\right)}\right) d \theta \\
& +\ell_{2} \lambda_{2} \chi\left(s_{2}, p_{2}\right) e^{-\mu_{2} \tau_{2}} \int_{0}^{\tau_{2}} F\left(\frac{\chi(s(t-\theta), p(t-\theta))}{\chi\left(s_{2}, p_{2}\right)}\right) d \theta \\
& +\ell_{3} \lambda_{3} \chi\left(s_{2}, p_{2}\right) e^{-\mu_{3} \tau_{3}} \int_{0}^{\tau_{3}} F\left(\frac{\chi(s(t-\theta), p(t-\theta))}{\chi\left(s_{2}, p_{2}\right)}\right) d \theta \\
& +\ell_{4}\left(p-p_{2}-\int_{p_{2}}^{p} \frac{g_{4}\left(p_{2}\right)}{g_{4}(\eta)} d \eta\right)+\ell_{5}\left(x-x_{2}-\int_{x_{2}}^{x} \frac{g_{5}\left(x_{2}\right)}{g_{5}(\eta)} d \eta\right) .
\end{aligned}
$$

Calculating $\frac{d V_{2}}{d t}$ along the solutions of model (12)-(17), we get

$$
\begin{aligned}
\frac{d V_{2}}{d t}= & \left(1-\frac{\chi\left(s_{2}, p_{2}\right)}{\chi\left(s, p_{2}\right)}\right)(\pi(s)-\lambda \chi(s, p)) \\
& +\ell_{1}\left(1-\frac{g_{1}\left(w_{2}\right)}{g_{1}(w)}\right)\left(\lambda_{1} e^{-\mu_{1} \tau_{1}} \chi\left(s\left(t-\tau_{1}\right), p\left(t-\tau_{1}\right)\right)-\left(a_{1}+\beta_{2}\right) g_{1}(w)\right) \\
& +\ell_{2}\left(1-\frac{g_{2}\left(y_{2}\right)}{g_{2}(y)}\right)\left(\lambda_{2} e^{-\mu_{2} \tau_{2}} \chi\left(s\left(t-\tau_{2}\right), p\left(t-\tau_{2}\right)\right)+a_{1} g_{1}(w)-\beta_{3} g_{2}(y)\right) \\
& +\ell_{3}\left(1-\frac{g_{3}\left(u_{2}\right)}{g_{3}(u)}\right)\left(\lambda_{3} e^{-\mu_{3} \tau_{3}} \chi\left(s\left(t-\tau_{3}\right), p\left(t-\tau_{3}\right)\right)-\beta_{4} g_{3}(u)\right) \\
& +\ell_{1} \lambda_{1} e^{-\mu_{1} \tau_{1}}\left(\chi(s, p)-\chi\left(s\left(t-\tau_{1}\right), p\left(t-\tau_{1}\right)\right)\right) \\
& +\ell_{1} \lambda_{1} e^{-\mu_{1} \tau_{1}} \chi\left(s_{2}, p_{2}\right) \ln \left(\frac{\chi\left(s\left(t-\tau_{1}\right), p\left(t-\tau_{1}\right)\right)}{\chi(s, p)}\right) \\
& +\ell_{2} \lambda_{2} e^{-\mu_{2} \tau_{2}}\left(\chi(s, p)-\chi\left(s\left(t-\tau_{2}\right), p\left(t-\tau_{2}\right)\right)\right)
\end{aligned}
$$




$$
\begin{aligned}
& +\ell_{2} \lambda_{2} e^{-\mu_{2} \tau_{2}} \chi\left(s_{2}, p_{2}\right) \ln \left(\frac{\chi\left(s\left(t-\tau_{2}\right), p\left(t-\tau_{2}\right)\right)}{\chi(s, p)}\right) \\
& +\ell_{3} \lambda_{3} e^{-\mu_{3} \tau_{3}}\left(\chi(s, p)-\chi\left(s\left(t-\tau_{3}\right), p\left(t-\tau_{3}\right)\right)\right) \\
& +\ell_{3} \lambda_{3} e^{-\mu_{3} \tau_{3}} \chi\left(s_{2}, p_{2}\right) \ln \left(\frac{\chi\left(s\left(t-\tau_{3}\right), p\left(t-\tau_{3}\right)\right)}{\chi(s, p)}\right) \\
& +\ell_{4}\left(1-\frac{g_{4}\left(p_{2}\right)}{g_{4}(p)}\right)\left(N \beta_{3} g_{2}(y)+M \beta_{4} g_{3}(u)-\beta_{5} g_{4}(p)-q g_{4}(p) g_{5}(x)\right) \\
& +\ell_{5}\left(1-\frac{g_{5}\left(x_{2}\right)}{g_{5}(x)}\right)\left(r g_{4}(p) g_{5}(x)-\beta_{6} g_{5}(x)\right) .
\end{aligned}
$$

Collecting terms of Eq. (40) and using the steady state conditions for $\Pi_{2}$ :

$$
\begin{aligned}
& \pi\left(s_{2}\right)=\lambda \chi\left(s_{2}, p_{2}\right), \\
& \left(a_{1}+\beta_{2}\right) g_{1}\left(w_{2}\right)=\lambda_{1} e^{-\mu_{1} \tau_{1}} \chi\left(s_{2}, p_{2}\right), \\
& \ell_{2} \beta_{3} g_{2}\left(y_{2}\right)=\left(\ell_{1} \lambda_{1} e^{-\mu_{1} \tau_{1}}+\ell_{2} \lambda_{2} e^{-\mu_{2} \tau_{2}}\right) \chi\left(s_{2}, p_{2}\right), \\
& \beta_{4} g_{3}\left(u_{2}\right)=\lambda_{3} e^{-\mu_{3} \tau_{3}} \chi\left(s_{2}, p_{2}\right), \quad \ell_{4} \beta_{5} g_{4}\left(p_{2}\right)=\lambda \chi\left(s_{2}, p_{2}\right)-q \ell_{4} g_{4}\left(p_{2}\right) g_{5}\left(x_{2}\right), \\
& \ell_{4} \beta_{5} g_{4}(p)=\lambda \chi\left(s_{2}, p_{2}\right) \frac{g_{4}(p)}{g_{4}\left(p_{2}\right)}-q \ell_{4} g_{4}(p) g_{5}\left(x_{2}\right),
\end{aligned}
$$

we obtain

$$
\begin{aligned}
\frac{d V_{2}}{d t}= & \left(\pi(s)-\pi\left(s_{2}\right)\right)\left(1-\frac{\chi\left(s_{2}, p_{2}\right)}{\chi\left(s, p_{2}\right)}\right)+\lambda \chi\left(s_{2}, p_{2}\right)\left(1-\frac{\chi\left(s_{2}, p_{2}\right)}{\chi\left(s, p_{2}\right)}\right) \\
& +\lambda \chi\left(s_{2}, p_{2}\right)\left(\frac{\chi(s, p)}{\chi\left(s, p_{2}\right)}-\frac{g_{4}(p)}{g_{4}\left(p_{2}\right)}\right) \\
& -\ell_{1} \lambda_{1} e^{-\mu_{1} \tau_{1}} \chi\left(s_{2}, p_{2}\right) \frac{g_{1}\left(w_{2}\right) \chi\left(s\left(t-\tau_{1}\right), p\left(t-\tau_{1}\right)\right)}{g_{1}(w) \chi\left(s_{2}, p_{2}\right)}+\ell_{1} \lambda_{1} e^{-\mu_{1} \tau_{1}} \chi\left(s_{2}, p_{2}\right) \\
& -\ell_{2} \lambda_{2} e^{-\mu_{2} \tau_{2}} \chi\left(s_{2}, p_{2}\right) \frac{g_{2}\left(y_{2}\right) \chi\left(s\left(t-\tau_{2}\right), p\left(t-\tau_{2}\right)\right)}{g_{2}(y) \chi\left(s_{2}, p_{2}\right)} \\
& -\ell_{1} \lambda_{1} e^{-\mu_{1} \tau_{1}} \chi\left(s_{2}, p_{2}\right) \frac{g_{2}\left(y_{2}\right) g_{1}(w)}{g_{2}(y) g_{1}\left(w_{2}\right)} \\
& +\left(\ell_{1} \lambda_{1} e^{-\mu_{1} \tau_{1}}+\ell_{2} \lambda_{2} e^{-\mu_{2} \tau_{2}}\right) \chi\left(s_{2}, p_{2}\right) \\
& -\ell_{3} \lambda_{3} e^{-\mu_{3} \tau_{3}} \chi\left(s_{2}, p_{2}\right) \frac{g_{3}\left(u_{2}\right) \chi\left(s\left(t-\tau_{3}\right), p\left(t-\tau_{3}\right)\right)}{g_{3}(u) \chi\left(s_{2}, p_{2}\right)} \\
& +\ell_{3} \lambda_{3} e^{-\mu_{3} \tau_{3}} \chi\left(s_{2}, p_{2}\right)+\ell_{1} \lambda_{1} e^{-\mu_{1} \tau_{1}} \chi\left(s_{2}, p_{2}\right) \ln \left(\frac{\chi\left(s\left(t-\tau_{1}\right), p\left(t-\tau_{1}\right)\right)}{\chi(s, p)}\right) \\
& -\left(\ell_{1} \lambda_{1} e^{-\mu_{1} \tau_{1}}+\ell_{2} \lambda_{2} e^{-\mu_{2} \tau_{2}}\right) \chi\left(s_{2}, p_{2}\right) \frac{g_{2}(y) g_{4}\left(p_{2}\right)}{g_{2}\left(y_{2}\right) g_{4}(p)} \\
& +\ell_{2} \lambda_{2} e^{-\mu_{2} \tau_{2}} \chi\left(s_{2}, p_{2}\right) \ln \left(\frac{\chi\left(s\left(t-\tau_{2}\right), p\left(t-\tau_{2}\right)\right)}{\chi(s, p)}\right) \\
& +\ell_{3} \lambda_{3} e^{-\mu_{3} \tau_{3}} \chi\left(s_{2}, p_{2}\right) \ln \left(\frac{\chi\left(s\left(t-\tau_{3}\right), p\left(t-\tau_{3}\right)\right)}{\mu_{3} \tau_{3}} \chi\left(s_{2}, p_{2}\right) \frac{g_{3}(u) g_{4}\left(p_{2}\right)}{g_{3}\left(u_{2}\right) g_{4}(p)}+\lambda \chi\left(s_{2}, p_{2}\right) .\right. \\
& \\
&
\end{aligned}
$$


By inequalities (30) with $\hat{s}=s_{2}, \hat{w}=w_{2}, \hat{y}=y_{2}$, and $\hat{p}=p_{2}$, we can get

$$
\begin{aligned}
\frac{d V_{2}}{d t}= & \left(\pi(s)-\pi\left(s_{2}\right)\right)\left(1-\frac{\chi\left(s_{2}, p_{2}\right)}{\chi\left(s, p_{2}\right)}\right)+\lambda \chi\left(s_{2}, p_{2}\right)\left(\frac{\chi(s, p)}{\chi\left(s, p_{2}\right)}-\frac{g_{4}(p)}{g_{4}\left(p_{2}\right)}\right)\left(1-\frac{\chi\left(s, p_{2}\right)}{\chi(s, p)}\right) \\
& -\lambda \chi\left(s_{2}, p_{2}\right)\left[F\left(\frac{\chi\left(s_{2}, p_{2}\right)}{\chi\left(s, p_{2}\right)}\right)+F\left(\frac{g_{4}(p) \chi\left(s, p_{2}\right)}{g_{4}\left(p_{2}\right) \chi(s, p)}\right)\right] \\
& -\ell_{1} \lambda_{1} e^{-\mu_{1} \tau_{1}} \chi\left(s_{2}, p_{2}\right)\left[F\left(\frac{g_{1}\left(w_{2}\right) \chi\left(s\left(t-\tau_{1}\right), p\left(t-\tau_{1}\right)\right)}{g_{1}(w) \chi\left(s_{2}, p_{2}\right)}\right)+F\left(\frac{g_{2}\left(y_{2}\right) g_{1}(w)}{g_{2}(y) g_{1}\left(w_{2}\right)}\right)\right] \\
& -\ell_{2} \lambda_{2} e^{-\mu_{2} \tau_{2}} \chi\left(s_{2}, p_{2}\right) F\left(\frac{g_{2}\left(y_{2}\right) \chi\left(s\left(t-\tau_{2}\right), p\left(t-\tau_{2}\right)\right)}{g_{2}(y) \chi\left(s_{2}, p_{2}\right)}\right) \\
& -\ell_{3} \lambda_{3} e^{-\mu_{3} \tau_{3}} \chi\left(s_{2}, p_{2}\right)\left[F\left(\frac{g_{3}\left(u_{2}\right) \chi\left(s\left(t-\tau_{3}\right), p\left(t-\tau_{3}\right)\right)}{g_{3}(u) \chi\left(s_{2}, p_{2}\right)}\right)+F\left(\frac{g_{3}(u) g_{4}\left(p_{2}\right)}{g_{3}\left(u_{2}\right) g_{4}(p)}\right)\right] \\
& -\left(\ell_{1} \lambda_{1} e^{-\mu_{1} \tau_{1}}+\ell_{2} \lambda_{2} e^{-\mu_{2} \tau_{2}}\right) \chi\left(s_{2}, p_{2}\right) F\left(\frac{g_{2}(y) g_{4}\left(p_{2}\right)}{g_{2}\left(y_{2}\right) g_{4}(p)}\right) .
\end{aligned}
$$

According to hypotheses (H1), (H2), and (H4), we get $\frac{d V_{2}}{d t} \leq 0$ and $\frac{d V_{2}}{d t}=0$ at $\Pi_{2}$. LIP implies that $\Pi_{2}$ is GAS.

\section{Model with delay-distributed}

In the next model, we consider a general delay-distributed HIV infection model with humoral immunity as follows:

$$
\begin{aligned}
& \dot{s}=\pi(s(t))-\lambda \chi(s(t), p(t)) \\
& \dot{w}=\lambda_{1} \int_{0}^{h_{1}} f_{1}(\tau) e^{-\mu_{1} \tau} \chi(s(t-\tau), p(t-\tau)) d \tau-\left(a_{1}+\beta_{2}\right) g_{1}(w(t)) \\
& \dot{y}=\lambda_{2} \int_{0}^{h_{2}} f_{2}(\tau) e^{-\mu_{2} \tau} \chi(s(t-\tau), p(t-\tau)) d \tau+a_{1} g_{1}(w(t))-\beta_{3} g_{2}(y(t)) \\
& \dot{u}=\lambda_{3} \int_{0}^{h_{3}} f_{3}(\tau) e^{-\mu_{3} \tau} \chi(s(t-\tau), p(t-\tau)) d \tau-\beta_{4} g_{3}(u(t)) \\
& \dot{p}=N \beta_{3} g_{2}(y(t))+M \beta_{4} g_{3}(u(t))-\beta_{5} g_{4}(p(t))-q g_{4}(p(t)) g_{5}(x(t)) \\
& \dot{x}=r g_{4}(p(t)) g_{5}(x(t))-\beta_{6} g_{5}(x(t))
\end{aligned}
$$

where $f_{i}(\tau) e^{-\mu_{i} \tau}$ over the time interval $\left[0, h_{i}\right], i=1,2,3$, represent the probabilities that uninfected cells contacted by HIV at time $t-\tau$ survived $\tau$ time units and became infected at time $t$.

The probability distribution function $f_{i}(\tau)$ is assumed to satisfy $f_{i}(\tau)>0$ and

$$
\int_{0}^{h_{i}} f_{i}(\tau) d \tau=1, \quad \int_{0}^{h_{i}} f_{i}(\eta) e^{v \eta} d \eta<\infty, \quad i=1,2,3
$$

where $v$ is a positive constant. Let us denote $\Theta_{i}(\tau)=f_{i}(\tau) e^{-\mu_{i} \tau}$ and $F_{i}=\int_{0}^{h_{i}} \Theta_{i}(\tau) d \tau$; thus $0<F_{i} \leq 1, i=1,2,3$.

Lemma 4 Let hypotheses (H1)-(H3) be valid, then the solutions of system (42)-(47) are nonnegative and ultimately bounded. 
Proof The nonnegativity of solutions of system (42)-(47) can easily be shown as given in Lemma 1.

From Eq. (42) we have that $\lim \sup _{t \rightarrow \infty} s(t) \leq \frac{b}{b}=M_{1}$. Let

$$
\begin{aligned}
G(t)= & N \int_{0}^{h_{1}} \Theta_{1}(\tau) s(t-\tau) d \tau+N \int_{0}^{h_{2}} \Theta_{2}(\tau) s(t-\tau) d \tau+M \int_{0}^{h_{3}} \Theta_{3}(\tau) s(t-\tau) d \tau \\
& +N w(t)+N y(t)+M u(t)+\frac{1}{2} p(t)+\frac{q}{2 r} x(t),
\end{aligned}
$$

then

$$
\begin{aligned}
& \dot{G}(t)=N \int_{0}^{h_{1}} \Theta_{1}(\tau)[\pi(s(t-\tau))-\lambda \chi(s(t-\tau), p(t-\tau))] d \tau \\
& +N \int_{0}^{h_{2}} \Theta_{2}(\tau)[\pi(s(t-\tau))-\lambda \chi(s(t-\tau), p(t-\tau))] d \tau \\
& +M \int_{0}^{h_{3}} \Theta_{3}(\tau)[\pi(s(t-\tau))-\lambda \chi(s(t-\tau), p(t-\tau))] d \tau \\
& +N\left[\lambda_{1} \int_{0}^{h_{1}} \Theta_{1}(\tau) \chi(s(t-\tau), p(t-\tau)) d \tau-\left(a_{1}+\beta_{2}\right) g_{1}(w(t))\right] \\
& +N\left[\lambda_{2} \int_{0}^{h_{2}} \Theta_{2}(\tau) \chi(s(t-\tau), p(t-\tau)) d \tau+a_{1} g_{1}(w(t))-\beta_{3} g_{2}(y(t))\right] \\
& +M\left[\lambda_{3} \int_{0}^{h_{3}} \Theta_{3}(\tau) \chi(s(t-\tau), p(t-\tau)) d \tau-\beta_{4} g_{3}(u(t))\right] \\
& +\frac{1}{2}\left[N \beta_{3} g_{2}(y(t))+M \beta_{4} g_{3}(u(t))-\beta_{5} g_{4}(p(t))-q g_{4}(p(t)) g_{5}(x(t))\right] \\
& +\frac{q}{2 r}\left[r g_{4}(p(t)) g_{5}(x(t))-\beta_{6} g_{5}(x(t))\right] \\
& \leq N \int_{0}^{h_{1}} \Theta_{1}(\tau)[b-\bar{b} s(t-\tau)] d \tau+N \int_{0}^{h_{2}} \Theta_{2}(\tau)[b-\bar{b} s(t-\tau)] d \tau \\
& +M \int_{0}^{h_{3}} \Theta_{3}(\tau)[b-\bar{b} s(t-\tau)] d \tau \\
& -N \beta_{2} \alpha_{1} w(t)-\frac{1}{2} N \beta_{3} \alpha_{2} y(t)-\frac{1}{2} M \beta_{4} \alpha_{3} u(t)-\frac{1}{2} \beta_{5} \alpha_{4} p(t)-\frac{q}{2 r} \beta_{6} \alpha_{5} x(t) \\
& \leq b\left(N F_{1}+N F_{2}+M F_{3}\right)-\sigma\left[N \int_{0}^{h_{1}} \Theta_{1}(\tau) s(t-\tau) d \tau+N \int_{0}^{h_{2}} \Theta_{2}(\tau) s(t-\tau) d \tau\right. \\
& \left.+M \int_{0}^{h_{3}} \Theta_{3}(\tau) s(t-\tau) d \tau+N w(t)+N y(t)+M u(t)+\frac{1}{2} p(t)+\frac{q}{2 r} x(t)\right] \\
& \leq b(2 N+M)-\sigma G(t) .
\end{aligned}
$$

Hence, $\lim \sup _{t \rightarrow \infty} G(t) \leq \frac{b(2 N+M)}{\sigma}$ and

$$
\begin{array}{ll}
\lim \sup _{t \rightarrow \infty} w(t) \leq M_{2}, & \quad \limsup _{t \rightarrow \infty} y(t) \leq M_{2}, \quad \\
\lim \sup _{t \rightarrow \infty} p(t) \leq M_{4}, & \lim \sup _{t \rightarrow \infty} u(t) \leq M_{3}, \\
\sup _{t \rightarrow \infty} x(t) \leq M_{5}, &
\end{array}
$$


where $\sigma, M_{1}, \ldots, M_{5}$ are given in the previous section. Therefore, $s(t), w(t), y(t), u(t), p(t)$, and $x(t)$ are ultimately bounded. Moreover, the set $\Omega$ defined by (19) is also positively invariant with respect to system (42)-(47).

\subsection{Steady states}

Lemma 5 Suppose that hypotheses (H1)-(H4) are valid (42)-(47), then there exist two bifurcation parameters $R_{0}$ and $R_{1}$ with $R_{0}>R_{1}>0$ such that

(i) if $R_{0} \leq 1$, then there exists only one steady state $\Pi_{0}$;

(ii) if $R_{1} \leq 1<R_{0}$, then there exist two steady states $\Pi_{0}$ and $\Pi_{1}$;

(iii) if $R_{1}>1$, then there exist three steady states $\Pi_{0}, \Pi_{1}$, and $\Pi_{2}$.

Proof Let $\Pi(s, w, y, u, p, x)$ be any steady state of (42)-(47) satisfying the following equations:

$$
\begin{aligned}
& 0=\pi(s)-\lambda \chi(s, p), \\
& 0=\lambda_{1} F_{1} \chi(s, p)-\left(a_{1}+\beta_{2}\right) g_{1}(w), \\
& 0=\lambda_{2} F_{2} \chi(s, p)+a_{1} g_{1}(w)-\beta_{3} g_{2}(y), \\
& 0=\lambda_{3} F_{3} \chi(s, p)-\beta_{4} g_{3}(u), \\
& 0=N \beta_{3} g_{2}(y)+M \beta_{4} g_{3}(u)-\beta_{5} g_{4}(p)-q g_{4}(p) g_{5}(x), \\
& 0=r g_{4}(p) g_{5}(x)-\beta_{6} g_{5}(x) .
\end{aligned}
$$

From Eq. (53) we obtain two possible solutions: $g_{5}(x)=0$ and $g_{4}(p)=\beta_{6} / r$. First, we consider the case $g_{5}(x)=0$, then from hypothesis (H3) we have $x=0$. Hypothesis (H3) implies that $g_{i}^{-1}, i=1, \ldots, 5$, exist, strictly increasing and $g_{i}^{-1}(0)=0$. Let us define

$$
\begin{aligned}
& \alpha_{1}(s)=g_{1}^{-1}\left(\frac{\lambda_{1} F_{1}}{\lambda\left(a_{1}+\beta_{2}\right)} \pi(s)\right), \quad \alpha_{2}(s)=g_{2}^{-1}\left(\frac{a_{1} \lambda_{1} F_{1}+\left(a_{1}+\beta_{2}\right) \lambda_{2} F_{2}}{\beta_{3} \lambda\left(a_{1}+\beta_{2}\right)} \pi(s)\right), \\
& \alpha_{3}(s)=g_{3}^{-1}\left(\frac{\lambda_{3} F_{3}}{\beta_{4} \lambda} \pi(s)\right), \quad \alpha_{4}(s)=g_{4}^{-1}\left(\frac{\zeta}{\lambda} \pi(s)\right),
\end{aligned}
$$

where

$$
\zeta=\frac{N\left(a_{1} \lambda_{1} F_{1}+\left(a_{1}+\beta_{2}\right) \lambda_{2} F_{2}\right)+M \lambda_{3}\left(a_{1}+\beta_{2}\right) F_{3}}{\beta_{5}\left(a_{1}+\beta_{2}\right)} .
$$

From Eqs. (48)-(53) we can get

$$
w=\alpha_{1}(s), \quad y=\alpha_{2}(s), \quad u=\alpha_{3}(s), \quad p=\alpha_{4}(s) .
$$

Obviously, $\alpha_{i}(s)>0$ for $s \in\left[0, s_{0}\right)$ and $\alpha_{i}\left(s_{0}\right)=0, i=1, \ldots, 4$. From Eqs. (48), (54), and (55) we obtain

$$
\zeta \chi\left(s, \alpha_{4}(s)\right)-g_{4}\left(\alpha_{4}(s)\right)=0
$$


Equation (56) admits a solution $s=s_{0}$ which gives the infection-free steady state $\Pi_{0}\left(s_{0}, 0\right.$, $0,0,0,0)$. Let

$$
\Psi_{1}(s)=\zeta \chi\left(s, \alpha_{4}(s)\right)-g_{4}\left(\alpha_{4}(s)\right)=0
$$

By hypotheses (H1) and (H2) we have $\Psi_{1}(0)=-g_{4}\left(\alpha_{4}(0)\right)<0$ and $\Psi_{1}\left(s_{0}\right)=0$. Moreover,

$$
\Psi_{1}^{\prime}\left(s_{0}\right)=\zeta\left[\frac{\partial \chi\left(s_{0}, 0\right)}{\partial s}+\alpha_{4}^{\prime}\left(s_{0}\right) \frac{\partial \chi\left(s_{0}, 0\right)}{\partial p}\right]-g_{4}^{\prime}(0) \alpha_{4}^{\prime}\left(s_{0}\right) .
$$

From hypothesis $(\mathrm{H} 2)$ we note that $\frac{\partial \chi\left(s_{0}, 0\right)}{\partial s}=0$. Then

$$
\Psi_{1}^{\prime}\left(s_{0}\right)=\alpha_{4}^{\prime}\left(s_{0}\right) g_{4}^{\prime}(0)\left(\frac{\zeta}{g_{4}^{\prime}(0)} \frac{\partial \chi\left(s_{0}, 0\right)}{\partial p}-1\right)
$$

From Eq. (54), we get

$$
\Psi_{1}^{\prime}\left(s_{0}\right)=\frac{\zeta}{\lambda} \pi^{\prime}\left(s_{0}\right)\left(\frac{\zeta}{g_{4}^{\prime}(0)} \frac{\partial \chi\left(s_{0}, 0\right)}{\partial p}-1\right) .
$$

Therefore, using hypothesis $(\mathrm{H} 1)$, we get $\pi^{\prime}\left(s_{0}\right)<0$. So that, if $\frac{\zeta}{g_{4}^{\prime}(0)} \frac{\partial \chi\left(s_{0}, 0\right)}{\partial p}>1$, then $\Psi_{1}^{\prime}\left(s_{0}\right)<$ 0 and there exists $s_{1} \in\left(0, s_{0}\right)$ such that $\Psi_{1}\left(s_{1}\right)=0$. Hypotheses (H1)-(H3) imply that

$$
w_{1}=\alpha_{1}\left(s_{1}\right)>0, \quad y_{1}=\alpha_{2}\left(s_{1}\right)>0, \quad u_{1}=\alpha_{3}\left(s_{1}\right)>0, \quad p_{1}=\alpha_{4}\left(s_{1}\right)>0 .
$$

It means that a humoral-inactivated infection steady state $\Pi_{1}\left(s_{1}, w_{1}, y_{1}, u_{1}, p_{1}, 0\right)$ exists when $\frac{\zeta}{g_{4}^{\prime}(0)} \frac{\partial \chi\left(s_{0}, 0\right)}{\partial p}>1$. Now we can define

$$
R_{0}=\frac{\zeta}{g_{4}^{\prime}(0)} \frac{\partial \chi\left(s_{0}, 0\right)}{\partial p}
$$

The other solution of Eq. (53) is $g_{4}\left(p_{2}\right)=\frac{\beta_{6}}{r}$, which yields $p_{2}=g_{4}^{-1}\left(\frac{\beta_{6}}{r}\right)>0$. Substitute $p=p_{2}$ in Eq. (48) and let $\Psi_{2}(s)=\pi(s)-\lambda \zeta\left(s, p_{2}\right)=0$. According to hypotheses (H1) and (H2), $\Psi_{2}$ is strictly decreasing, $\Psi_{2}(0)=\pi(0)>0$, and $\Psi_{2}\left(s_{0}\right)=-\lambda \zeta\left(s_{0}, p_{2}\right)<0$. Thus, there exists a unique $s_{2} \in\left(0, s_{0}\right)$ such that $\Psi_{2}\left(s_{2}\right)=0$. It follows from Eqs. (52) and (55) that

$$
\begin{aligned}
& w_{2}=\alpha_{1}\left(s_{2}\right)>0, \quad y_{2}=\alpha_{2}\left(s_{2}\right)>0, \quad u_{2}=\alpha_{3}\left(s_{2}\right)>0, \quad p_{2}=g_{4}^{-1}\left(\frac{\beta_{6}}{r}\right)>0, \\
& x_{2}=g_{5}^{-1}\left(\frac{\beta_{5}}{q}\left(\zeta \frac{\chi\left(s_{2}, p_{2}\right)}{g_{4}\left(p_{2}\right)}-1\right)\right) .
\end{aligned}
$$

Thus, $x_{2}>0$ when $\zeta \frac{\chi\left(s_{2}, p_{2}\right)}{g_{4}\left(p_{2}\right)}>1$. Let us define the parameter $R_{1}$ as follows:

$$
R_{1}=\zeta \frac{\chi\left(s_{2}, p_{2}\right)}{g_{4}\left(p_{2}\right)}
$$

If $R_{1}>1$, then $x_{2}=g_{5}^{-1}\left(\frac{\beta_{5}}{q}\left(R_{1}-1\right)\right)>0$, and there exists a humoral-activated infection steady state $\Pi_{2}\left(s_{2}, w_{2}, y_{2}, u_{2}, p_{2}, x_{2}\right)$. 
Clearly, from hypotheses (H2) and (H4), we have

$$
R_{1}=\zeta \frac{\chi\left(s_{2}, p_{2}\right)}{g_{4}\left(p_{2}\right)} \leq \zeta \lim _{p \rightarrow 0^{+}} \frac{\chi\left(s_{2}, p\right)}{g_{4}(p)}=\frac{\zeta}{g_{4}^{\prime}(0)} \frac{\partial \chi\left(s_{2}, 0\right)}{\partial p}<\frac{\zeta}{g_{4}^{\prime}(0)} \frac{\partial \chi\left(s_{0}, 0\right)}{\partial p}=R_{0} .
$$

\subsection{Global properties}

Theorem 4 If $R_{0} \leq 1$ and hypotheses $(\mathrm{H} 1)-(\mathrm{H} 4)$ hold true for system (42)-(47), then $\Pi_{0}$ is GAS.

Proof Define

$$
\begin{aligned}
W_{0}= & s-s_{0}-\int_{s_{0}}^{s} \lim _{p \rightarrow 0^{+}} \frac{\chi\left(s_{0}, p\right)}{\chi(\eta, p)} d \eta+\kappa_{1} w+\kappa_{2} y+\kappa_{3} u \\
& +\kappa_{1} \lambda_{1} \int_{0}^{h_{1}} \Theta_{1}(\tau) \int_{0}^{\tau} \chi(s(t-\theta), p(t-\theta)) d \theta d \tau \\
& +\kappa_{2} \lambda_{2} \int_{0}^{h_{2}} \Theta_{2}(\tau) \int_{0}^{\tau} \chi(s(t-\theta), p(t-\theta)) d \theta d \tau \\
& +\kappa_{3} \lambda_{3} \int_{0}^{h_{3}} \Theta_{3}(\tau) \int_{0}^{\tau} \chi(s(t-\theta), p(t-\theta)) d \theta d \tau \\
& +\kappa_{4} p+\kappa_{5} x,
\end{aligned}
$$

where

$$
\begin{aligned}
& \lambda=\lambda_{1} \kappa_{1} F_{1}+\lambda_{2} \kappa_{2} F_{2}+\lambda_{3} \kappa_{3} F_{3}, \quad\left(a_{1}+\beta_{2}\right) \kappa_{1}=a_{1} \kappa_{2}, \\
& \kappa_{2}=N \kappa_{4}, \quad \kappa_{3}=M \kappa_{4}, \quad q \kappa_{4}=r \kappa_{5} .
\end{aligned}
$$

The solution of Eqs. (59) is given by

$$
\kappa_{1}=\frac{a_{1} N \lambda}{\zeta \beta_{5}\left(a_{1}+\beta_{2}\right)}, \quad \kappa_{2}=\frac{N \lambda}{\zeta \beta_{5}}, \quad \kappa_{3}=\frac{M \lambda}{\zeta \beta_{5}}, \quad \kappa_{4}=\frac{\lambda}{\zeta \beta_{5}}, \quad \kappa_{5}=\frac{q \lambda}{r \zeta \beta_{5}} .
$$

We evaluate $\frac{d W_{0}}{d t}$ along the solutions of (42)-(47) as follows:

$$
\begin{aligned}
\frac{d W_{0}}{d t}= & \left(1-\lim _{p \rightarrow 0^{+}} \frac{\chi\left(s_{0}, p\right)}{\chi(s, p)}\right)(\pi(s)-\lambda \chi(s, p))+\kappa_{1} \lambda_{1} \int_{0}^{h_{1}} \Theta_{1}(\tau) \chi(s(t-\tau), p(t-\tau)) d \tau \\
& -\kappa_{1}\left(a_{1}+\beta_{2}\right) g_{1}(w)+\kappa_{2} \lambda_{2} \int_{0}^{h_{2}} \Theta_{2}(\tau) \chi(s(t-\tau), p(t-\tau)) d \tau+a_{1} \kappa_{2} g_{1}(w) \\
& -\kappa_{2} \beta_{3} g_{2}(y)+\kappa_{3} \lambda_{3} \int_{0}^{h_{3}} \Theta_{3}(\tau) \chi(s(t-\tau), p(t-\tau)) d \tau-\kappa_{3} \beta_{4} g_{3}(u) \\
& +\kappa_{1} \lambda_{1} \int_{0}^{h_{1}} \Theta_{1}(\tau)(\chi(s, p)-\chi(s(t-\tau), p(t-\tau))) d \tau \\
& +\kappa_{2} \lambda_{2} \int_{0}^{h_{2}} \Theta_{2}(\tau)(\chi(s, p)-\chi(s(t-\tau), p(t-\tau))) d \tau \\
& +\kappa_{3} \lambda_{3} \int_{0}^{h_{3}} \Theta_{3}(\tau)(\chi(s, p)-\chi(s(t-\tau), p(t-\tau))) d \tau
\end{aligned}
$$




$$
\begin{aligned}
& +\kappa_{4}\left(N \beta_{3} g_{2}(y)+M \beta_{4} g_{3}(u)-\beta_{5} g_{4}(p)-q g_{4}(p) g_{5}(x)\right) \\
& +\kappa_{5}\left(r g_{4}(p) g_{5}(x)-\beta_{6} g_{5}(x)\right) .
\end{aligned}
$$

Collecting terms of Eq. (61) and using $\pi\left(s_{0}\right)=0$, we obtain

$$
\begin{aligned}
\frac{d W_{0}}{d t}= & \left(\pi(s)-\pi\left(s_{0}\right)\right)\left(1-\lim _{p \rightarrow 0^{+}} \frac{\chi\left(s_{0}, p\right)}{\chi(s, p)}\right) \\
& +\left(\frac{\lambda \chi(s, p)}{g_{4}(p)} \lim _{p \rightarrow 0^{+}} \frac{\chi\left(s_{0}, p\right)}{\chi(s, p)}-\kappa_{4} \beta_{5}\right) g_{4}(p)-\kappa_{5} \beta_{6} g_{5}(x) \\
\leq & \left(\pi(s)-\pi\left(s_{0}\right)\right)\left(1-\lim _{p \rightarrow 0^{+}} \frac{\chi\left(s_{0}, p\right)}{\chi(s, p)}\right) \\
& +\left(\lim _{p \rightarrow 0^{+}} \frac{\lambda \chi(s, p)}{g_{4}(p)} \lim _{p \rightarrow 0^{+}} \frac{\chi\left(s_{0}, p\right)}{\chi(s, p)}-\kappa_{4} \beta_{5}\right) g_{4}(p)-\kappa_{5} \beta_{6} g_{5}(x) \\
= & \left(\pi(s)-\pi\left(s_{0}\right)\right)\left(1-\frac{\partial \chi\left(s_{0}, 0\right) / \partial p}{\partial \chi(s, 0) / \partial p}\right) \\
& +\kappa_{4} \beta_{5}\left(\frac{\lambda}{\kappa_{4} \beta_{5} g_{4}^{\prime}(0)} \frac{\partial \chi\left(s_{0}, 0\right)}{\partial p}-1\right) g_{4}(p)-\kappa_{5} \beta_{6} g_{5}(x) \\
= & \left(\pi(s)-\pi\left(s_{0}\right)\right)\left(1-\frac{\partial \chi\left(s_{0}, 0\right) / \partial p}{\partial \chi(s, 0) / \partial p}\right)+\kappa_{4} \beta_{5}\left(R_{0}-1\right) g_{4}(p)-\kappa_{5} \beta_{6} g_{5}(x) .
\end{aligned}
$$

From hypotheses (H1) and (H2), we have

$$
\left(\pi(s)-\pi\left(s_{0}\right)\right)\left(1-\frac{\partial \chi\left(s_{0}, 0\right) / \partial p}{\partial \chi(s, 0) / \partial p}\right) \leq 0 .
$$

Therefore, if $R_{0} \leq 1$, then $\frac{d W_{0}}{d t} \leq 0$. Thus, $\Pi_{0}$ is GAS.

Theorem 5 If $R_{1} \leq 1<R_{0}$ and hypotheses (H1)-(H4) are valid for (42)-(47), then $\Pi_{1}$ is GAS.

Proof We introduce

$$
\begin{aligned}
W_{1}= & s-s_{1}-\int_{s_{1}}^{s} \frac{\chi\left(s_{1}, p_{1}\right)}{\chi\left(\eta, p_{1}\right)} d \eta+\kappa_{1}\left(w-w_{1}-\int_{w_{1}}^{w} \frac{g_{1}\left(w_{1}\right)}{g_{1}(\eta)} d \eta\right) \\
& +\kappa_{2}\left(y-y_{1}-\int_{y_{1}}^{y} \frac{g_{2}\left(y_{1}\right)}{g_{2}(\eta)} d \eta\right)+\kappa_{3}\left(u-u_{1}-\int_{u_{1}}^{u} \frac{g_{3}\left(u_{1}\right)}{g_{3}(\eta)} d \eta\right) \\
& +\kappa_{1} \lambda_{1} \chi\left(s_{1}, p_{1}\right) \int_{0}^{h_{1}} \Theta_{1}(\tau) \int_{0}^{\tau} F\left(\frac{\chi(s(t-\theta), p(t-\theta))}{\chi\left(s_{1}, p_{1}\right)}\right) d \theta d \tau \\
& +\kappa_{2} \lambda_{2} \chi\left(s_{1}, p_{1}\right) \int_{0}^{h_{2}} \Theta_{2}(\tau) \int_{0}^{\tau} F\left(\frac{\chi(s(t-\theta), p(t-\theta))}{\chi\left(s_{1}, p_{1}\right)}\right) d \theta d \tau \\
& +\kappa_{3} \lambda_{3} \chi\left(s_{1}, p_{1}\right) \int_{0}^{h_{3}} \Theta_{3}(\tau) \int_{0}^{\tau} F\left(\frac{\chi(s(t-\theta), p(t-\theta))}{\chi\left(s_{1}, p_{1}\right)}\right) d \theta d \tau \\
& +\kappa_{4}\left(p-p_{1}-\int_{p_{1}}^{p} \frac{g_{4}\left(p_{1}\right)}{g_{4}(\eta)} d \eta\right)+\kappa_{5} x .
\end{aligned}
$$


Evaluating $\frac{d W_{1}}{d t}$ along the trajectories of (42)-(47), we have

$$
\begin{aligned}
\frac{d W_{1}}{d t}= & \left(1-\frac{\chi\left(s_{1}, p_{1}\right)}{\chi\left(s, p_{1}\right)}\right)(\pi(s)-\lambda \chi(s, p)) \\
& +\kappa_{1}\left(1-\frac{g_{1}\left(w_{1}\right)}{g_{1}(w)}\right)\left(\lambda_{1} \int_{0}^{h_{1}} \Theta_{1}(\tau) \chi(s(t-\tau), p(t-\tau)) d \tau-\left(a_{1}+\beta_{2}\right) g_{1}(w)\right) \\
& +\kappa_{2}\left(1-\frac{g_{2}\left(y_{1}\right)}{g_{2}(y)}\right)\left(\lambda_{2} \int_{0}^{h_{2}} \Theta_{2}(\tau) \chi(s(t-\tau), p(t-\tau)) d \tau+a_{1} g_{1}(w)-\beta_{3} g_{2}(y)\right) \\
& +\kappa_{3}\left(1-\frac{g_{3}\left(u_{1}\right)}{g_{3}(u)}\right)\left(\lambda_{3} \int_{0}^{h_{3}} \Theta_{3}(\tau) \chi(s(t-\tau), p(t-\tau)) d \tau-\beta_{4} g_{3}(u)\right) \\
& +\kappa_{1} \lambda_{1} \int_{0}^{h_{1}} \Theta_{1}(\tau)\left(\chi(s, p)-\chi(s(t-\tau), p(t-\tau))+\chi\left(s_{1}, p_{1}\right)\right. \\
& \left.\times \ln \left(\frac{\chi(s(t-\tau), p(t-\tau))}{\chi(s, p)}\right)\right) d \tau \\
& +\kappa_{2} \lambda_{2} \int_{0}^{h_{2}} \Theta_{2}(\tau)\left(\chi(s, p)-\chi(s(t-\tau), p(t-\tau))+\chi\left(s_{1}, p_{1}\right)\right. \\
& +\kappa_{4}\left(1-\frac{g_{4}\left(p_{1}\right)}{g_{4}(p)}\right)\left(N g_{4}(p) g_{5}(x)-\beta_{6} g_{5}(x)\right) . \\
& \times \ln \left(\frac{\chi(s(t-\tau), p(t-\tau))}{\chi(s, p)}\right) d \tau \\
& +\kappa_{3} \lambda_{3} \int_{0}^{h_{3}} \Theta_{3}(\tau)\left(\chi(s, p)-\chi(s(t-\tau), p(t-\tau))+\chi\left(s_{1}, p_{1}\right)\right. \\
& (s(t-\tau), p(t-\tau)) \\
\chi & (s, p)) d \tau
\end{aligned}
$$

Collecting terms of Eq. (63) and applying the conditions of the steady state $\Pi_{1}$ :

$$
\begin{aligned}
& \pi\left(s_{1}\right)=\lambda \chi\left(s_{1}, p_{1}\right), \\
& \left(a_{1}+\beta_{2}\right) g_{1}\left(w_{1}\right)=\lambda_{1} F_{1} \chi\left(s_{1}, p_{1}\right), \quad \kappa_{2} \beta_{3} g_{2}\left(y_{1}\right)=\left(\kappa_{1} \lambda_{1} F_{1}+\kappa_{2} \lambda_{2} F_{2}\right) \chi\left(s_{1}, p_{1}\right), \\
& \beta_{4} g_{3}\left(u_{1}\right)=\lambda_{3} F_{3} \chi\left(s_{1}, p_{1}\right), \quad \kappa_{4} \beta_{5} g_{4}\left(p_{1}\right)=\lambda \chi\left(s_{1}, p_{1}\right),
\end{aligned}
$$

we get

$$
\begin{aligned}
\frac{d W_{1}}{d t}= & \left(\pi(s)-\pi\left(s_{1}\right)\right)\left(1-\frac{\chi\left(s_{1}, p_{1}\right)}{\chi\left(s, p_{1}\right)}\right) \\
& +\lambda \chi\left(s_{1}, p_{1}\right)\left(1-\frac{\chi\left(s_{1}, p_{1}\right)}{\chi\left(s, p_{1}\right)}\right)+\lambda \chi\left(s_{1}, p_{1}\right)\left(\frac{\chi(s, p)}{\chi\left(s, p_{1}\right)}-\frac{g_{4}(p)}{g_{4}\left(p_{1}\right)}\right) \\
& -\kappa_{1} \lambda_{1} \chi\left(s_{1}, p_{1}\right) \int_{0}^{h_{1}} \Theta_{1}(\tau) \frac{\chi(s(t-\tau), p(t-\tau)) g_{1}\left(w_{1}\right)}{\chi\left(s_{1}, p_{1}\right) g_{1}(w)} d \tau+\kappa_{1} \lambda_{1} F_{1} \chi\left(s_{1}, p_{1}\right) \\
& -\kappa_{2} \lambda_{2} \chi\left(s_{1}, p_{1}\right) \int_{0}^{h_{2}} \Theta_{2}(\tau) \frac{\chi(s(t-\tau), p(t-\tau)) g_{2}\left(y_{1}\right)}{\chi\left(s_{1}, p_{1}\right) g_{2}(y)} d \tau
\end{aligned}
$$




$$
\begin{aligned}
& -\kappa_{1} \lambda_{1} F_{1} \chi\left(s_{1}, p_{1}\right) \frac{g_{2}\left(y_{1}\right) g_{1}(w)}{g_{2}(y) g_{1}\left(w_{1}\right)}+\left(\kappa_{1} \lambda_{1} F_{1}+\kappa_{2} \lambda_{2} F_{2}\right) \chi\left(s_{1}, p_{1}\right) \\
& -\kappa_{3} \lambda_{3} \chi\left(s_{1}, p_{1}\right) \int_{0}^{h_{3}} \Theta_{3}(\tau) \frac{\chi\left(s(t-\tau), p(t-\tau) g_{3}\left(u_{1}\right)\right)}{\chi\left(s_{1}, p_{1}\right) g_{3}(u)} d \tau+\kappa_{3} \lambda_{3} F_{3} \chi\left(s_{1}, p_{1}\right) \\
& +\kappa_{1} \lambda_{1} \chi\left(s_{1}, p_{1}\right) \int_{0}^{h_{1}} \Theta_{1}(\tau) \ln \left(\frac{\chi(s(t-\tau), p(t-\tau))}{\chi(s, p)}\right) d \tau \\
& +\kappa_{2} \lambda_{2} \chi\left(s_{1}, p_{1}\right) \int_{0}^{h_{2}} \Theta_{2}(\tau) \ln \left(\frac{\chi(s(t-\tau), p(t-\tau))}{\chi(s, p)}\right) d \tau \\
& +\kappa_{3} \lambda_{3} \chi\left(s_{1}, p_{1}\right) \int_{0}^{h_{3}} \Theta_{3}(\tau) \ln \left(\frac{\chi(s(t-\tau), p(t-\tau))}{\chi(s, p)}\right) d \tau \\
& -\left(\kappa_{1} \lambda_{1} F_{1}+\kappa_{2} \lambda_{2} F_{2}\right) \chi\left(s_{1}, p_{1}\right) \frac{g_{2}(y) g_{4}\left(p_{1}\right)}{g_{2}\left(y_{1}\right) g_{4}(p)}-\kappa_{3} \lambda_{3} F_{3} \chi\left(s_{1}, p_{1}\right) \frac{g_{3}(u) g_{4}\left(p_{1}\right)}{g_{3}\left(u_{1}\right) g_{4}(p)} \\
& +\lambda \chi\left(s_{1}, p_{1}\right)+r \kappa_{5}\left(g_{4}\left(p_{1}\right)-\frac{\beta_{6}}{r}\right) g_{5}(x) .
\end{aligned}
$$

Using inequalities (30) with $\hat{s}=s_{1}, \hat{w}=w_{1}, \hat{y}=y_{1}, \hat{p}=p_{1}$, and $\tau_{1}=\tau_{2}=\tau_{3}=\tau$, we can obtain

$$
\begin{aligned}
\frac{d W_{1}}{d t}= & \left(\pi(s)-\pi\left(s_{1}\right)\right)\left(1-\frac{\chi\left(s_{1}, p_{1}\right)}{\chi\left(s, p_{1}\right)}\right) \\
& +\lambda \chi\left(s_{1}, p_{1}\right)\left(\frac{\chi(s, p)}{\chi\left(s, p_{1}\right)}-\frac{g_{4}(p)}{g_{4}\left(p_{1}\right)}\right)\left(1-\frac{\chi\left(s, p_{1}\right)}{\chi(s, p)}\right) \\
& -\lambda \chi\left(s_{1}, p_{1}\right)\left[F\left(\frac{\chi\left(s_{1}, p_{1}\right)}{\chi\left(s, p_{1}\right)}\right)+F\left(\frac{g_{4}(p) \chi\left(s, p_{1}\right)}{g_{4}\left(p_{1}\right) \chi(s, p)}\right)\right] \\
& -\kappa_{1} \lambda_{1} \chi\left(s_{1}, p_{1}\right) \int_{0}^{h_{1}} \Theta_{1}(\tau) F\left(\frac{g_{1}\left(w_{1}\right) \chi(s(t-\tau), p(t-\tau))}{g_{1}(w) \chi\left(s_{1}, p_{1}\right)}\right) d \tau \\
& -\kappa_{2} \lambda_{2} \chi\left(s_{1}, p_{1}\right) \int_{0}^{h_{2}} \Theta_{2}(\tau) F\left(\frac{g_{2}\left(y_{1}\right) \chi(s(t-\tau), p(t-\tau))}{g_{2}(y) \chi\left(s_{1}, p_{1}\right)}\right) d \tau \\
& -\kappa_{3} \lambda_{3} \chi\left(s_{1}, p_{1}\right) \int_{0}^{h_{3}} \Theta_{3}(\tau) F\left(\frac{g_{3}\left(u_{1}\right) \chi(s(t-\tau), p(t-\tau))}{g_{3}(u) \chi\left(s_{1}, p_{1}\right)}\right) \\
& -\kappa_{1} \lambda_{1} F_{1} \chi\left(s_{1}, p_{1}\right) F\left(\frac{g_{2}\left(y_{1}\right) g_{1}(w)}{g_{2}(y) g_{1}\left(w_{1}\right)}\right) \\
& -\left(\kappa_{1} \lambda_{1} F_{1}+\kappa_{2} \lambda_{2} F_{2}\right) \chi\left(s_{1}, p_{1}\right) F\left(\frac{g_{2}(y) g_{4}\left(p_{1}\right)}{g_{2}\left(y_{1}\right) g_{4}(p)}\right) \\
& -\kappa_{3} \lambda_{3} F_{3} \chi\left(s_{1}, p_{1}\right) F\left(\frac{g_{3}(u) g_{4}\left(p_{1}\right)}{g_{3}\left(u_{1}\right) g_{4}(p)}\right)+r \kappa_{5}\left(g_{4}\left(p_{1}\right)-g_{4}\left(p_{2}\right)\right) g_{5}(x) .
\end{aligned}
$$

Using hypotheses (H1), (H2), (H4), and Lemma 3, we get $\frac{d W_{1}}{d t} \leq 0$, where the equality occurs at $\Pi_{1}$. By LIP, $\Pi_{1}$ is GAS.

Theorem 6 If $R_{1}>1$ and hypotheses $(\mathrm{H} 1)-(\mathrm{H} 4)$ are valid for (42)-(47), then $\Pi_{2}$ is GAS.

Proof Define

$$
W_{2}=s-s_{2}-\int_{s_{2}}^{s} \frac{\chi\left(s_{2}, p_{2}\right)}{\chi\left(\eta, p_{2}\right)} d \eta+\kappa_{1}\left(w-w_{2}-\int_{w_{2}}^{w} \frac{g_{1}\left(w_{2}\right)}{g_{1}(\eta)} d \eta\right)
$$




$$
\begin{aligned}
& +\kappa_{2}\left(y-y_{2}-\int_{y_{2}}^{y} \frac{g_{2}\left(y_{2}\right)}{g_{2}(\eta)} d \eta\right)+\kappa_{3}\left(u-u_{2}-\int_{u_{2}}^{u} \frac{g_{3}\left(u_{2}\right)}{g_{3}(\eta)} d \eta\right) \\
& +\kappa_{1} \lambda_{1} \chi\left(s_{2}, p_{2}\right) \int_{0}^{h_{1}} \Theta_{1}(\tau) \int_{0}^{\tau} F\left(\frac{\chi(s(t-\theta), p(t-\theta))}{\chi\left(s_{2}, p_{2}\right)}\right) d \theta d \tau \\
& +\kappa_{2} \lambda_{2} \chi\left(s_{2}, p_{2}\right) \int_{0}^{h_{2}} \Theta_{2}(\tau) \int_{0}^{\tau} F\left(\frac{\chi(s(t-\theta), p(t-\theta))}{\chi\left(s_{2}, p_{2}\right)}\right) d \theta d \tau \\
& +\kappa_{3} \lambda_{3} \chi\left(s_{2}, p_{2}\right) \int_{0}^{h_{3}} \Theta_{3}(\tau) \int_{0}^{\tau} F\left(\frac{\chi(s(t-\theta), p(t-\theta))}{\chi\left(s_{2}, p_{2}\right)}\right) d \theta d \tau \\
& +\kappa_{4}\left(p-p_{2}-\int_{p_{2}}^{p} \frac{g_{4}\left(p_{2}\right)}{g_{4}(\eta)} d \eta\right)+\kappa_{5}\left(x-x_{2}-\int_{x_{2}}^{x} \frac{g_{5}\left(x_{2}\right)}{g_{5}(\eta)} d \eta\right) .
\end{aligned}
$$

Calculating $\frac{d W_{2}}{d t}$ along the solutions of model (42)-(47), we get

$$
\begin{aligned}
& \frac{d W_{2}}{d t}=\left(1-\frac{\chi\left(s_{2}, p_{2}\right)}{\chi\left(s, p_{2}\right)}\right)(\pi(s)-\lambda \chi(s, p)) \\
& +\kappa_{1}\left(1-\frac{g_{1}\left(w_{2}\right)}{g_{1}(w)}\right)\left(\lambda_{1} \int_{0}^{h_{1}} \Theta_{1}(\tau) \chi(s(t-\tau), p(t-\tau)) d \tau-\left(a_{1}+\beta_{2}\right) g_{1}(w)\right) \\
& +\kappa_{2}\left(1-\frac{g_{2}\left(y_{2}\right)}{g_{2}(y)}\right)\left(\lambda_{2} \int_{0}^{h_{2}} \Theta_{2}(\tau) \chi(s(t-\tau), p(t-\tau)) d \tau+a_{1} g_{1}(w)-\beta_{3} g_{2}(y)\right) \\
& +\kappa_{3}\left(1-\frac{g_{3}\left(u_{2}\right)}{g_{3}(u)}\right)\left(\lambda_{3} \int_{0}^{h_{3}} \Theta_{3}(\tau) \chi(s(t-\tau), p(t-\tau)) d \tau-\beta_{4} g_{3}(u)\right) \\
& +\kappa_{1} \lambda_{1} \int_{0}^{h_{1}} \Theta_{1}(\tau)\left(\chi(s, p)-\chi(s(t-\tau), p(t-\tau))+\chi\left(s_{2}, p_{2}\right)\right. \\
& \left.\times \ln \left(\frac{\chi(s(t-\tau), p(t-\tau))}{\chi(s, p)}\right)\right) d \tau \\
& +\kappa_{2} \lambda_{2} \int_{0}^{h_{2}} \Theta_{2}(\tau)\left(\chi(s, p)-\chi(s(t-\tau), p(t-\tau))+\chi\left(s_{2}, p_{2}\right)\right. \\
& \left.\times \ln \left(\frac{\chi(s(t-\tau), p(t-\tau))}{\chi(s, p)}\right)\right) d \tau \\
& +\kappa_{3} \lambda_{3} \int_{0}^{h_{3}} \Theta_{3}(\tau)\left(\chi(s, p)-\chi(s(t-\tau), p(t-\tau))+\chi\left(s_{2}, p_{2}\right)\right. \\
& \left.\times \ln \left(\frac{\chi(s(t-\tau), p(t-\tau))}{\chi(s, p)}\right)\right) d \tau \\
& +\kappa_{4}\left(1-\frac{g_{4}\left(p_{2}\right)}{g_{4}(p)}\right)\left(N \beta_{3} g_{2}(y)+M \beta_{4} g_{3}(u)-\beta_{5} g_{4}(p)-q g_{4}(p) g_{5}(x)\right) \\
& +\kappa_{5}\left(1-\frac{g_{5}\left(x_{2}\right)}{g_{5}(x)}\right)\left(r g_{4}(p) g_{5}(x)-\beta_{6} g_{5}(x)\right) \text {. }
\end{aligned}
$$

Collecting terms of Eq. (64) and applying the steady state conditions for $\Pi_{2}$ :

$$
\begin{aligned}
& \pi\left(s_{2}\right)=\lambda \chi\left(s_{2}, p_{2}\right), \\
& \left(a_{1}+\beta_{2}\right) g_{1}\left(w_{2}\right)=\lambda_{1} F_{1} \chi\left(s_{2}, p_{2}\right), \quad \kappa_{2} \beta_{3} g_{2}\left(y_{2}\right)=\left(\kappa_{1} \lambda_{1} F_{1}+\kappa_{2} \lambda_{2} F_{2}\right) \chi\left(s_{2}, p_{2}\right), \\
& \beta_{4} g_{3}\left(u_{2}\right)=\lambda_{3} F_{3} \chi\left(s_{2}, p_{2}\right), \quad \kappa_{4} \beta_{5} g_{4}\left(p_{2}\right)=\lambda \chi\left(s_{2}, p_{2}\right)-q \kappa_{4} g_{4}\left(p_{2}\right) g_{5}\left(x_{2}\right),
\end{aligned}
$$




$$
\kappa_{4} \beta_{5} g_{4}(p)=\lambda \chi\left(s_{2}, p_{2}\right) \frac{g_{4}(p)}{g_{4}\left(p_{2}\right)}-q \kappa_{4} g_{4}(p) g_{5}\left(x_{2}\right)
$$

we obtain

$$
\begin{aligned}
\frac{d W_{2}}{d t}= & \left(\pi(s)-\pi\left(s_{2}\right)\right)\left(1-\frac{\chi\left(s_{2}, p_{2}\right)}{\chi\left(s, p_{2}\right)}\right)+\lambda \chi\left(s_{2}, p_{2}\right)\left(1-\frac{\chi\left(s_{2}, p_{2}\right)}{\chi\left(s, p_{2}\right)}\right) \\
& +\lambda \chi\left(s_{2}, p_{2}\right)\left(\frac{\chi(s, p)}{\chi\left(s, p_{2}\right)}-\frac{g_{4}(p)}{g_{4}\left(p_{2}\right)}\right) \\
& -\kappa_{1} \lambda_{1} \chi\left(s_{2}, p_{2}\right) \int_{0}^{h_{1}} \Theta_{1}(\tau) \frac{g_{1}\left(w_{2}\right) \chi(s(t-\tau), p(t-\tau))}{g_{1}(w) \chi\left(s_{2}, p_{2}\right)} d \tau \\
& +\kappa_{1} \lambda_{1} F_{1} \chi\left(s_{2}, p_{2}\right)-\kappa_{2} \lambda_{2} \chi\left(s_{2}, p_{2}\right) \int_{0}^{h_{2}} \Theta_{2}(\tau) \frac{g_{2}\left(y_{2}\right) \chi(s(t-\tau), p(t-\tau))}{g_{2}(y) \chi\left(s_{2}, p_{2}\right)} d \tau \\
& -\kappa_{1} \lambda_{1} F_{1} \chi\left(s_{2}, p_{2}\right) \frac{g_{2}\left(y_{2}\right) g_{1}(w)}{g_{2}(y) g_{1}\left(w_{2}\right)}+\left(\kappa_{1} \lambda_{1} F_{1}+\kappa_{2} \lambda_{2} F_{2}\right) \chi\left(s_{2}, p_{2}\right) \\
& -\kappa_{3} \lambda_{3} \chi\left(s_{2}, p_{2}\right) \int_{0}^{h_{3}} \Theta_{3}(\tau) \frac{g_{3}\left(u_{2}\right) \chi(s(t-\tau), p(t-\tau))}{g_{3}(u) \chi\left(s_{2}, p_{2}\right)} d \tau+\kappa_{3} \lambda_{3} F_{3} \chi\left(s_{2}, p_{2}\right) \\
& +\kappa_{1} \lambda_{1} \chi\left(s_{2}, p_{2}\right) \int_{0}^{h_{1}} \Theta_{1}(\tau) \ln \left(\frac{\chi(s(t-\tau), p(t-\tau))}{\chi(s, p)}\right) d \tau \\
& +\kappa_{2} \lambda_{2} \chi\left(s_{2}, p_{2}\right) \int_{0}^{h_{2}} \Theta_{2}(\tau) \ln \left(\frac{\chi(s(t-\tau), p(t-\tau))}{\chi(s, p)}\right) d \tau \\
& +\kappa_{3} \lambda_{3} \chi\left(s_{2}, p_{2}\right) \int_{0}^{h_{3}} \Theta_{3}(\tau) \ln \left(\frac{\chi(s(t-\tau), p(t-\tau))}{\chi(s, p)}\right) d \tau \\
& -\left(\kappa_{1} \lambda_{1} F_{1}+\kappa_{2} \lambda_{2} F_{2}\right) \chi\left(s_{2}, p_{2}\right) \frac{g_{2}(y) g_{4}\left(p_{2}\right)}{g_{2}\left(y_{2}\right) g_{4}(p)} \\
& -\kappa_{3} \lambda_{3} F_{3} \chi\left(s_{2}, p_{2}\right) \frac{g_{3}(u) g_{4}\left(p_{2}\right)}{g_{3}\left(u_{2}\right) g_{4}(p)}+\lambda \chi\left(s_{2}, p_{2}\right) .
\end{aligned}
$$

Using inequalities (30) with $\hat{s}=s_{2}, \hat{w}=w_{2}, \hat{y}=y_{2}, \hat{p}=p_{2}$, and $\tau_{1}=\tau_{2}=\tau_{3}=\tau$, we can obtain

$$
\begin{aligned}
\frac{d W_{2}}{d t}= & \left(\pi(s)-\pi\left(s_{2}\right)\right)\left(1-\frac{\chi\left(s_{2}, p_{2}\right)}{\chi\left(s, p_{2}\right)}\right) \\
& +\lambda \chi\left(s_{2}, p_{2}\right)\left(\frac{\chi(s, p)}{\chi\left(s, p_{2}\right)}-\frac{g_{4}(p)}{g_{4}\left(p_{2}\right)}\right)\left(1-\frac{\chi\left(s, p_{2}\right)}{\chi(s, p)}\right) \\
& -\lambda \chi\left(s_{2}, p_{2}\right)\left[F\left(\frac{\chi\left(s_{2}, p_{2}\right)}{\chi\left(s, p_{2}\right)}\right)+F\left(\frac{g_{4}(p) \chi\left(s, p_{2}\right)}{g_{4}\left(p_{2}\right) \chi(s, p)}\right)\right] \\
& -\kappa_{1} \lambda_{1} \chi\left(s_{2}, p_{2}\right) \int_{0}^{h_{1}} \Theta_{1}(\tau) F\left(\frac{g_{1}\left(w_{2}\right) \chi(s(t-\tau), p(t-\tau))}{g_{1}(w) \chi\left(s_{2}, p_{2}\right)}\right) d \tau \\
& -\kappa_{2} \lambda_{2} \chi\left(s_{2}, p_{2}\right) \int_{0}^{h_{2}} \Theta_{2}(\tau) F\left(\frac{g_{2}\left(y_{2}\right) \chi(s(t-\tau), p(t-\tau))}{g_{2}(y) \chi\left(s_{2}, p_{2}\right)}\right) d \tau \\
& -\kappa_{3} \lambda_{3} \chi\left(s_{2}, p_{2}\right) \int_{0}^{h_{3}} \Theta_{3}(\tau) F\left(\frac{g_{3}\left(u_{2}\right) \chi(s(t-\tau), p(t-\tau))}{g_{3}(u) \chi\left(s_{2}, p_{2}\right)}\right) d \tau \\
& -\kappa_{1} \lambda_{1} F_{1} \chi\left(s_{2}, p_{2}\right) F\left(\frac{g_{2}\left(y_{2}\right) g_{1}(w)}{g_{2}(y) g_{1}\left(w_{2}\right)}\right)
\end{aligned}
$$




$$
\begin{aligned}
& -\left(\kappa_{1} \lambda_{1} F_{1}+\kappa_{2} \lambda_{2} F_{2}\right) \chi\left(s_{2}, p_{2}\right) F\left(\frac{g_{2}(y) g_{4}\left(p_{2}\right)}{g_{2}\left(y_{2}\right) g_{4}(p)}\right) \\
& -\kappa_{3} \lambda_{3} F_{3} \chi\left(s_{2}, p_{2}\right) F\left(\frac{g_{3}(u) g_{4}\left(p_{2}\right)}{g_{3}\left(u_{2}\right) g_{4}(p)}\right) .
\end{aligned}
$$

According to hypotheses (H1), (H2), and (H4), we get $\frac{d W_{2}}{d t} \leq 0$. Applying LIP, one can show that $\Pi_{2}$ is GAS.

\section{Numerical simulations}

We now perform some computer simulations on the following application:

$$
\begin{aligned}
& \dot{s}(t)=\rho-\beta_{1} s(t)+\omega s(t)\left(1-\frac{s(t)}{s_{\max }}\right)-\frac{\left(1-\varepsilon_{1}\right) \bar{\lambda} s(t) p(t)}{1+\eta p(t)}, \\
& \dot{w}(t)=\frac{\left(1-\varepsilon_{1}\right) \bar{\lambda}_{1} e^{-\mu_{1} \tau_{1}} s\left(t-\tau_{1}\right) p\left(t-\tau_{1}\right)}{1+\eta p\left(t-\tau_{1}\right)}-\left(a_{1}+\beta_{2}\right) w(t), \\
& \dot{y}(t)=\frac{\left(1-\varepsilon_{1}\right) \bar{\lambda}_{2} e^{-\mu_{2} \tau_{2}} s\left(t-\tau_{2}\right) p\left(t-\tau_{2}\right)}{1+\eta p\left(t-\tau_{2}\right)}+a_{1} w(t)-\beta_{3} y(t), \\
& \dot{u}(t)=\frac{\left(1-\varepsilon_{1}\right) \bar{\lambda}_{3} e^{-\mu_{3} \tau_{3}} s\left(t-\tau_{3}\right) p\left(t-\tau_{3}\right)}{1+\eta p\left(t-\tau_{3}\right)}-\beta_{4} u(t), \\
& \dot{p}(t)=\left(1-\varepsilon_{2}\right) \bar{N} \beta_{3} y(t)+\left(1-\varepsilon_{2}\right) \bar{M} \beta_{4} u(t)-\beta_{5} p(t)-q p(t) x(t), \\
& \dot{x}(t)=r p(t) x(t)-\beta_{6} x(t) .
\end{aligned}
$$

We assume that $\omega<\beta_{1}$. In this application, we consider the following specific forms of the general functions:

$$
\begin{aligned}
& \pi(s(t))=\rho-\beta_{1} s(t)+\omega s(t)\left(1-\frac{s(t)}{s_{\max }}\right), \quad \chi(s(t), p(t))=\frac{s(t) p(t)}{1+\eta p(t)}, \\
& g_{i}(\theta)=\theta, \quad i=1, \ldots, 5
\end{aligned}
$$

First we verify hypotheses $(\mathrm{H} 1)-(\mathrm{H} 4)$ for the chosen forms, then we solve the system using MATLAB. Clearly, $\pi(0)=\rho>0$ and $\pi\left(s_{0}\right)=0$, where

$$
s_{0}=\frac{s_{\max }}{2 \omega}\left(\omega-\beta_{1}+\sqrt{\left(\omega-\beta_{1}\right)^{2}+\frac{4 \rho \omega}{s_{\max }}}\right) .
$$

We have

$$
\pi^{\prime}(s)=-\beta_{1}+\omega-\frac{2 \omega s}{s_{\max }}<0 .
$$

Clearly, $\pi(s)>0$ for $s \in\left[0, s_{0}\right)$ and

$$
\pi(s)=\rho-\left(\beta_{1}-\omega\right) s-\omega \frac{s^{2}}{s_{\max }} \leq \rho-\left(\beta_{1}-\omega\right) s .
$$


Then hypothesis (H1) is satisfied. We also have $\chi(s, p)>0, \chi(0, p)=\chi(s, 0)=0$ for $s, p \in$ $(0, \infty)$, and

$$
\frac{\partial \chi(s, p)}{\partial s}=\frac{p}{1+\eta p}, \quad \frac{\partial \chi(s, p)}{\partial p}=\frac{s}{(1+\eta p)^{2}}, \quad \frac{\partial \chi(s, 0)}{\partial p}=s .
$$

Then $\frac{\partial \chi(s, p)}{\partial s}>0, \frac{\partial \chi(s, p)}{\partial p}>0$, and $\frac{\partial \chi(s, 0)}{\partial p}>0$ for $s, p \in(0, \infty)$. Therefore, hypothesis (H1) is satisfied. In addition,

$$
\begin{aligned}
& \chi(s, p)=\frac{s p}{1+\eta p} \leq s p=p \frac{\partial \chi(s, 0)}{\partial p}, \\
& \left(\frac{\partial \chi(s, 0)}{\partial p}\right)^{\prime}=1>0 \quad \text { for all } s>0 .
\end{aligned}
$$

It follows that (H2) is satisfied. Clearly, hypothesis (H3) holds true. Moreover,

$$
\frac{\partial}{\partial p}\left(\frac{\chi(s, p)}{g_{4}(p)}\right)=\frac{-\eta s}{(1+\eta p)^{2}}<0
$$

Therefore, hypothesis ( $\mathrm{H} 4)$ holds true and Theorems 1-3 are applicable. The parameters $R_{0}$ and $R_{1}$ for this application are given by

$$
\begin{aligned}
& R_{0}=\frac{\left(1-\varepsilon_{1}\right)\left(1-\varepsilon_{2}\right)\left\{\bar{N}\left(a_{1} \bar{\lambda}_{1} e^{-\mu_{1} \tau_{1}}+\left(a_{1}+\beta_{2}\right) \bar{\lambda}_{2} e^{-\mu_{2} \tau_{2}}\right)+\overline{M \lambda_{3}} e^{-\mu_{3} \tau_{3}}\left(a_{1}+\beta_{2}\right)\right\}}{\beta_{5}\left(a_{1}+\beta_{2}\right)} s_{0}, \\
& R_{1}=\frac{\left(1-\varepsilon_{1}\right)\left(1-\varepsilon_{2}\right)\left\{\bar{N}\left(a_{1} \bar{\lambda}_{1} e^{-\mu_{1} \tau_{1}}+\left(a_{1}+\beta_{2}\right) \bar{\lambda}_{2} e^{-\mu_{2} \tau_{2}}\right)+\overline{M \lambda}_{3} e^{-\mu_{3} \tau_{3}}\left(a_{1}+\beta_{2}\right)\right\}}{\beta_{5}\left(a_{1}+\beta_{2}\right)} \frac{s_{2}}{1+\eta p_{2}} .
\end{aligned}
$$

Remark There are several forms of the general function $\chi(s, p)$ where $(\mathrm{H} 1)-(\mathrm{H} 4)$ can be satisfied such as:

(i) Holling-type incidence $\chi(s, p)=\frac{s p}{1+\eta_{1} s}$,

(ii) Beddington-DeAngelis incidence $\chi(s, p)=\frac{s p}{1+\eta_{1} s+\eta_{2} p}$,

(iii) Crowley-Martin incidence $\chi(s, p)=\frac{s p}{\left(1+\eta_{1} s\right)\left(1+\eta_{2} p\right)}$,

(iv) Hill-type incidence $\chi(s, p)=\frac{s^{m} p}{\eta^{m}+s^{m}}$.

Now we are ready to perform some numerical simulations for system (66)-(71). The data of system (66)-(71) are provided in Table 1 . We let $\tau_{1}=\tau_{2}=\tau_{3}=\tau$.

-Effect of the parameters $\lambda_{i}$ and $r$ on the stability of the steady states

To discuss our global results, we choose three different initial conditions:

IC1: $(s(0), w(0), y(0), u(0), p(0), x(0))=(900,5,5,15,3,3)$.

Table 1 The data of example (66)-(71)

\begin{tabular}{ll}
\hline Parameter & Value \\
\hline$\rho$ & 10 \\
$\beta_{1}$ & 0.01 \\
$\omega$ & 0.0001 \\
$s_{\max }$ & 1200 \\
$q$ & 0.5 \\
\hline
\end{tabular}

\begin{tabular}{ll}
\hline Parameter & Value \\
\hline$\beta_{2}$ & 0.02 \\
$\beta_{3}$ & 0.36 \\
$\beta_{4}$ & 0.031 \\
$\beta_{5}$ & 3.0 \\
$\beta_{6}$ & 0.1 \\
\hline
\end{tabular}

\begin{tabular}{ll}
\hline Parameter & Value \\
\hline$\mu_{1}$ & 1 \\
$\mu_{2}$ & 1 \\
$\mu_{3}$ & 1 \\
$\eta$ & 0.01 \\
$a_{1}$ & 0.2 \\
\hline
\end{tabular}

\begin{tabular}{ll}
\hline Parameter & Value \\
$\overline{\bar{N}}$ & 6 \\
$\bar{M}$ & 3 \\
$\varepsilon_{1}, \varepsilon_{2}, \tau$ & Varied \\
$\lambda_{i}, i=1,2,3$ & Varied \\
$r$ & Varied \\
\hline
\end{tabular}




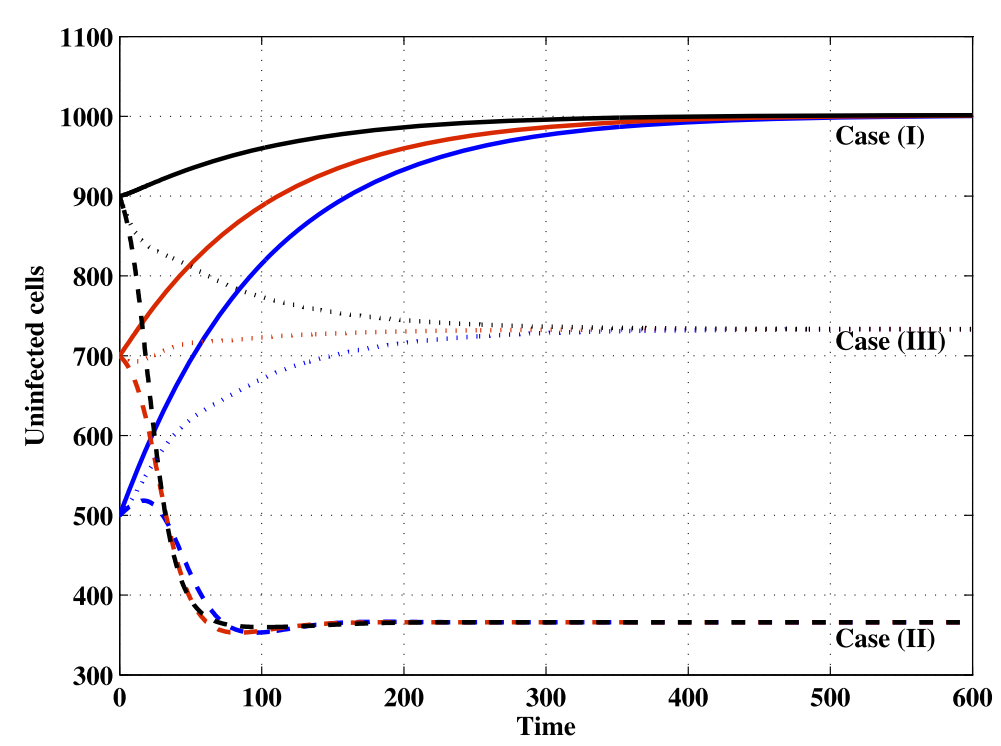

Figure 1 The concentration of uninfected CD4 ${ }^{+} \mathrm{T}$ cells for system (66)-(71)

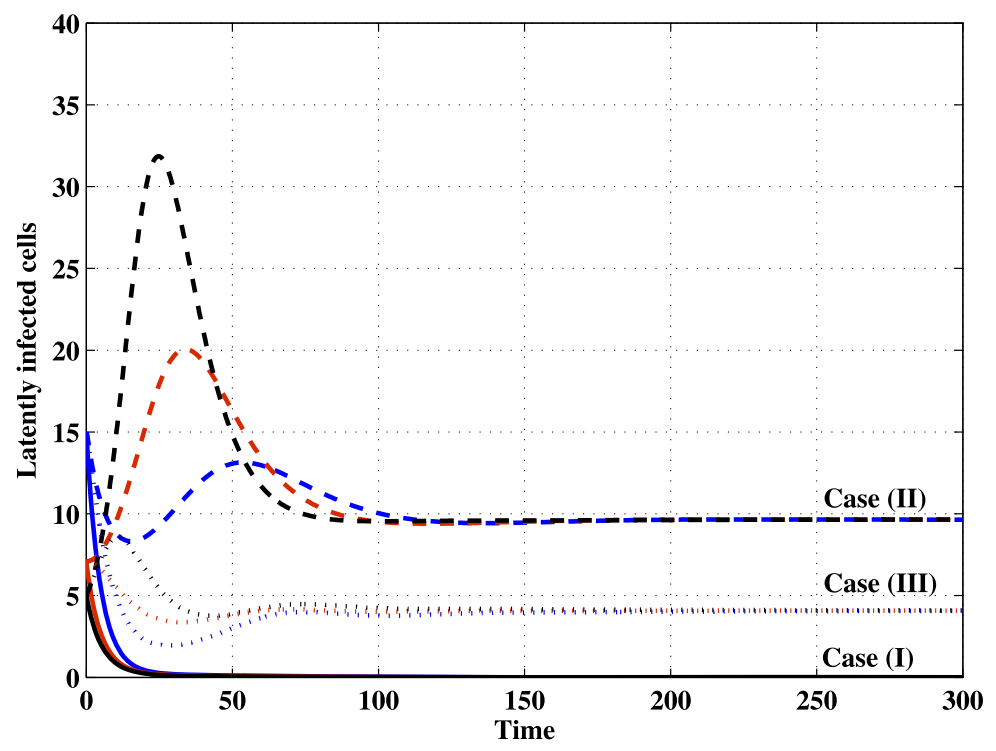

Figure 2 The concentration of latently infected cells for system (66)-(71)

IC2: $(s(0), w(0), y(0), u(0), p(0), x(0))=(700,7,8,30,5,5)$.

IC3: $(s(0), w(0), y(0), u(0), p(0), x(0))=(500,15,18,60,12,7)$.

Let us address three cases for the parameters $\lambda_{i}, i=1,2,3$, and $r$. We assume that $\varepsilon_{1}=$ $\varepsilon_{2}=0$ (there is no treatment) and $\tau_{i}=0$ (there is no time delay).

Case (I): Choose $\lambda_{i}=0.0000625$ and $r=0.005$, which gives $R_{0}=0.3016<1$ and $R_{1}=$ $0.1917<1$. Therefore, based on Lemma 2 and Theorem 1 , the system has unique steady state, that is, $\Pi_{0}$, and it is GAS. As we can see from Figs. 1-6, the concentration of the uninfected $\mathrm{CD} 4^{+} \mathrm{T}$ cells is increased and approaches its normal value before infection, 


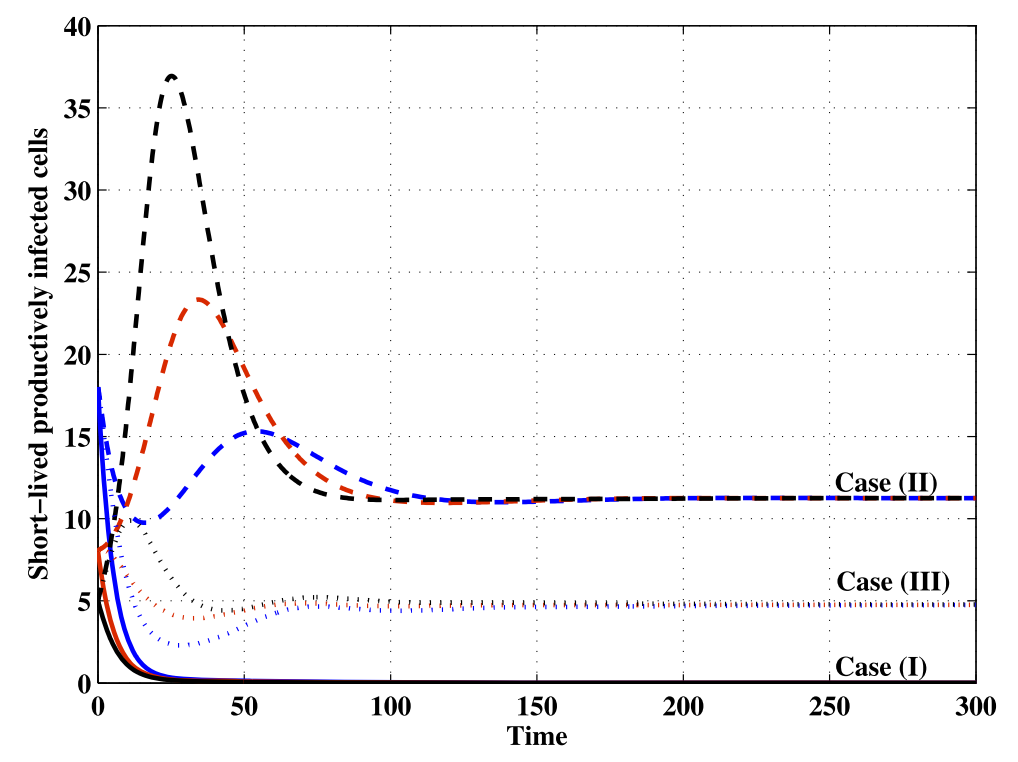

Figure 3 The concentration of short-lived productively infected cells for system (66)-(71)

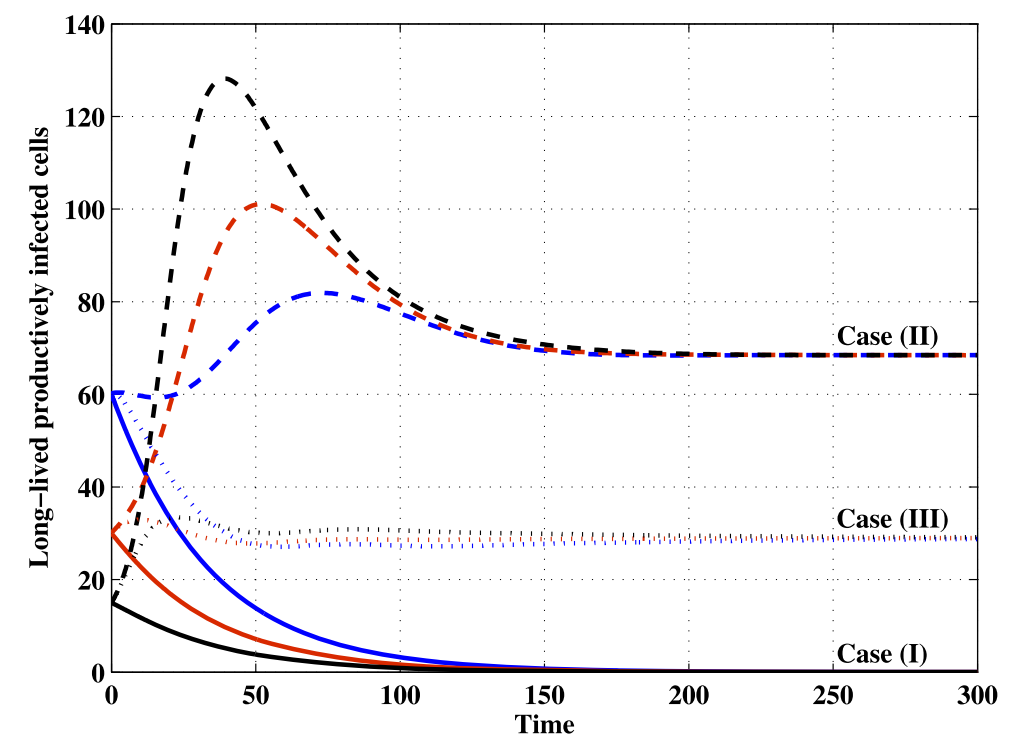

Figure 4 The concentration of long-lived productively infected cells for system (66)-(71)

that is, $s_{0}=1001.98$; while concentrations of the other compartments converge to zero for all the three initial conditions. As a result, the HIV-1 is removed from the plasma.

Case (II): By taking $\lambda_{i}=0.000625$ and $r=0.005$. For these values, $R_{1}=0.6095<1<R_{0}=$ 3.0163. Consequently, based on Lemma 2 and Theorem 2 , the humoral-inactivated infection steady state $\Pi_{1}$ is positive and is GAS. Figures 1-6 confirm that the numerical results support the theoretical results presented in Theorem 2. It can be observed that the variables of the model eventually converge to $\Pi_{1}=(366.317,9.72758,11.3488,69.0344$, 


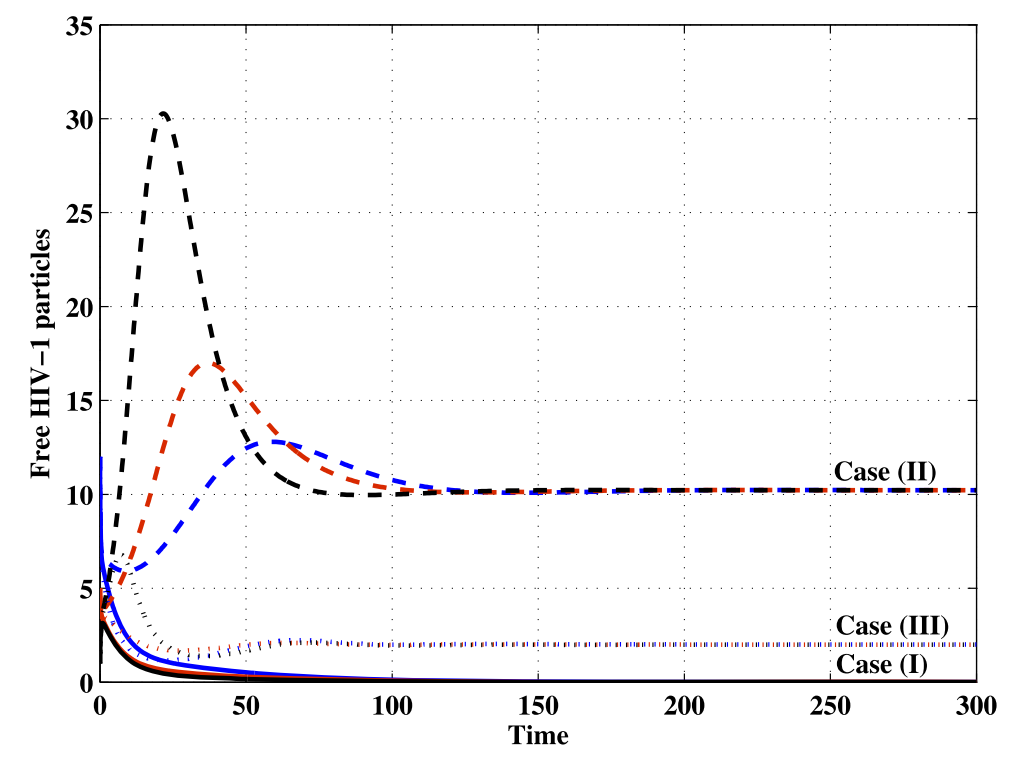

Figure 5 The concentration of free virus particles for system (66)-(71)

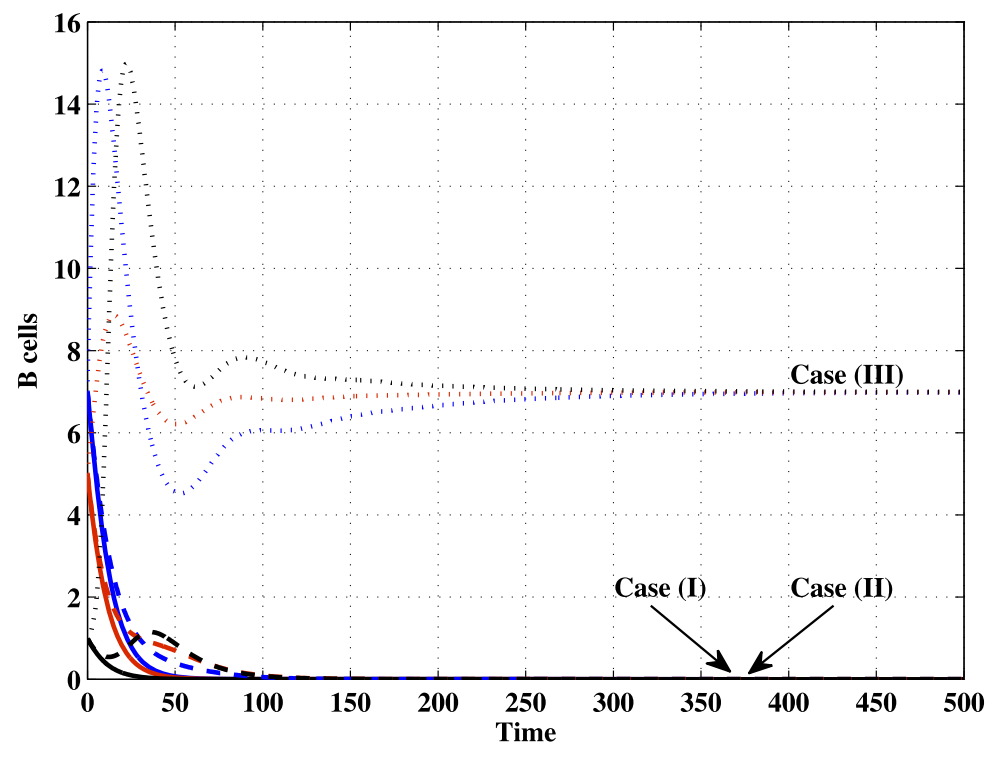

Figure 6 The concentration of B cells for system (66)-(71)

$10.3112,0.0)$ for all the three initial conditions. This case corresponds to a chronic HIV-1 infection in the absence of immune response.

Case (III): $\lambda_{i}=0.000625$ and $r=0.05$. Then we calculate $R_{0}=3.0163>1$ and $R_{1}=$ $2.1648>1$. According to Lemma 2 and Theorem 3, the humoral-activated infection steady state $\Pi_{2}$ is positive and is GAS. We can see from Figs. 1-6 that there is a consistency between the numerical results and theoretical results of Theorem 3 . The states of the system converge to $\Pi_{2}=(734.586,4.09194,4.77393,29.0396,2.0,7.01238)$ for all the three initial 


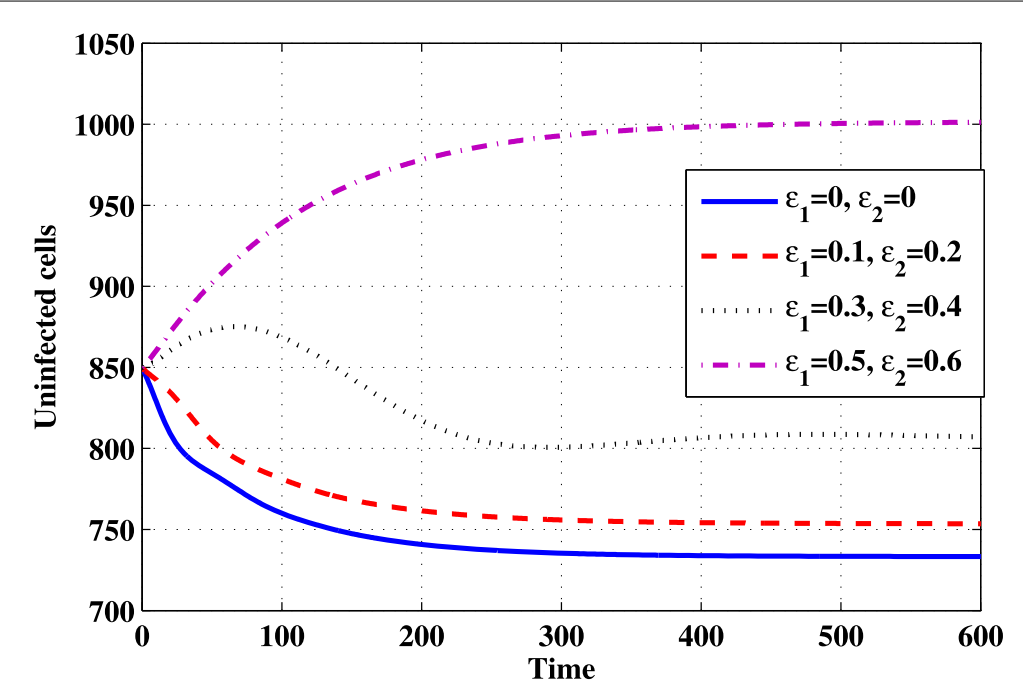

Figure 7 The concentration of uninfected $C D 4^{+} T$ cells for system (66)-(71)

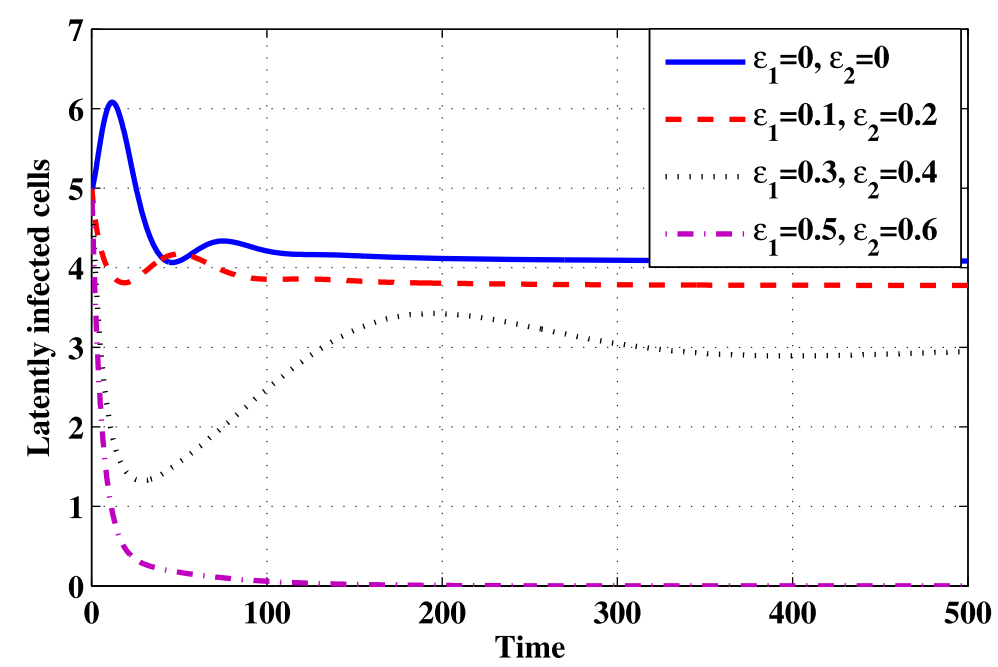

Figure 8 The concentration of latently infected cells for system (66)-(71)

conditions. In this case the humoral immune response is activated and can control the disease.

-Effect of the HAART on the HIV dynamics

We take $\tau_{i}=0, \lambda_{i}=0.000625$, and $r=0.05$. We choose the initial conditions $(s(0), w(0)$, $y(0), u(0), p(0), x(0))=(850,5,6,17,1.5,5)$. In Figs. 7-12 we show the effect of the drug efficacy parameters $\varepsilon_{1}$ and $\varepsilon_{2}$ on the HIV dynamics. Also, we can observe that, as the drug efficacy parameters $\varepsilon_{1}$ and $\varepsilon_{2}$ are increased, the concentration of uninfected cells is increased, while the concentrations of free virus particles and the three types of infected cells are decreased. Table 2 shows that the values of $R_{0}$ and $R_{1}$ are decreased as $\varepsilon_{1}$ and $\varepsilon_{2}$ are increased.

Let us define the overall HAART effect as $\varepsilon_{e}=\varepsilon_{1}+\varepsilon_{2}-\varepsilon_{1} \varepsilon_{2}$ [13]. If $\varepsilon_{e}=0$, then the HAART has no effect, if $\varepsilon_{e}=1$, the HIV growth is completely halted. Consequently, the 


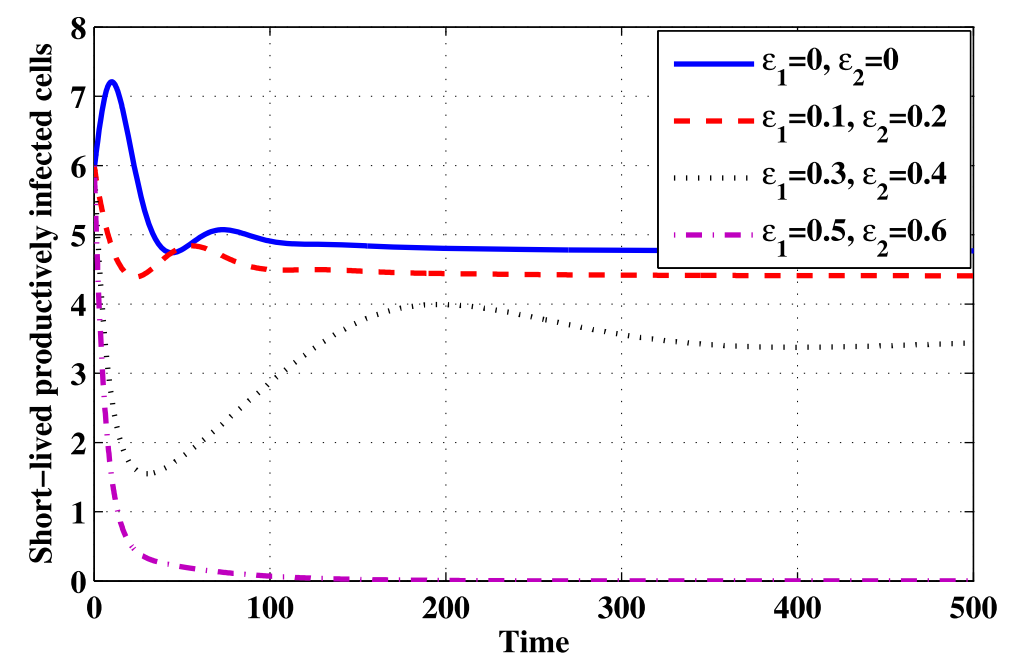

Figure 9 The concentration of short-lived productively infected cells for system (66)-(71)

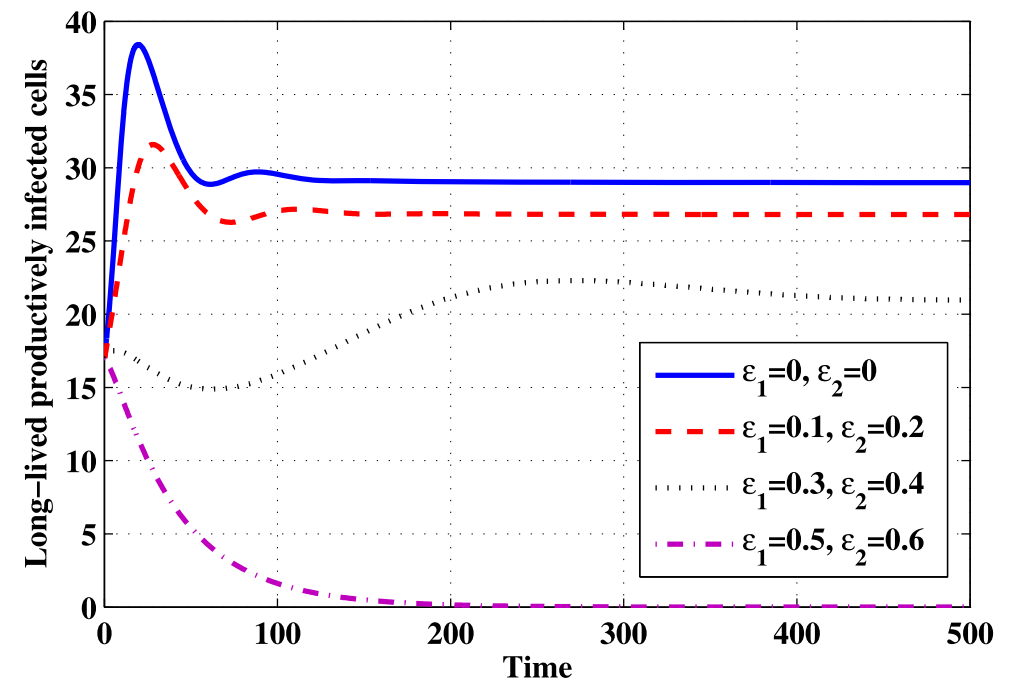

Figure 10 The concentration of long-lived productively infected cells for system (66)-(71)

parameter $R_{0}$ is given by

$$
R_{0}\left(\varepsilon_{e}\right)=\frac{\left(1-\varepsilon_{e}\right)\left\{\bar{N}\left(a_{1} \bar{\lambda}_{1} e^{-\mu_{1} \tau_{1}}+\left(a_{1}+\beta_{2}\right) \bar{\lambda}_{2} e^{-\mu_{2} \tau_{2}}\right)+\bar{M} \bar{\lambda}_{3} e^{-\mu_{3} \tau_{3}}\left(a_{1}+\beta_{2}\right)\right\}}{\beta_{5}\left(a_{1}+\beta_{2}\right)} s_{0} .
$$

Since the goal is to clear the viruses from the body, we have to determine the drug efficacy that makes $R_{0}\left(\varepsilon_{e}\right) \leq 1$ for system (66)-(71). In this case, we get the critical drug efficacy (i.e., the efficacy needed in order to stabilize the system around the uninfected steady state). For the model (66) $-(71), \Pi_{0}$ is GAS when $R_{0}\left(\varepsilon_{e}\right) \leq 1$, i.e.,

$$
\varepsilon_{e}^{\text {crit }} \leq \varepsilon_{e}<1, \quad \varepsilon_{e}^{\text {crit }}=\max \left\{0, \frac{R_{0}(0)-1}{R_{0}(0)}\right\} .
$$




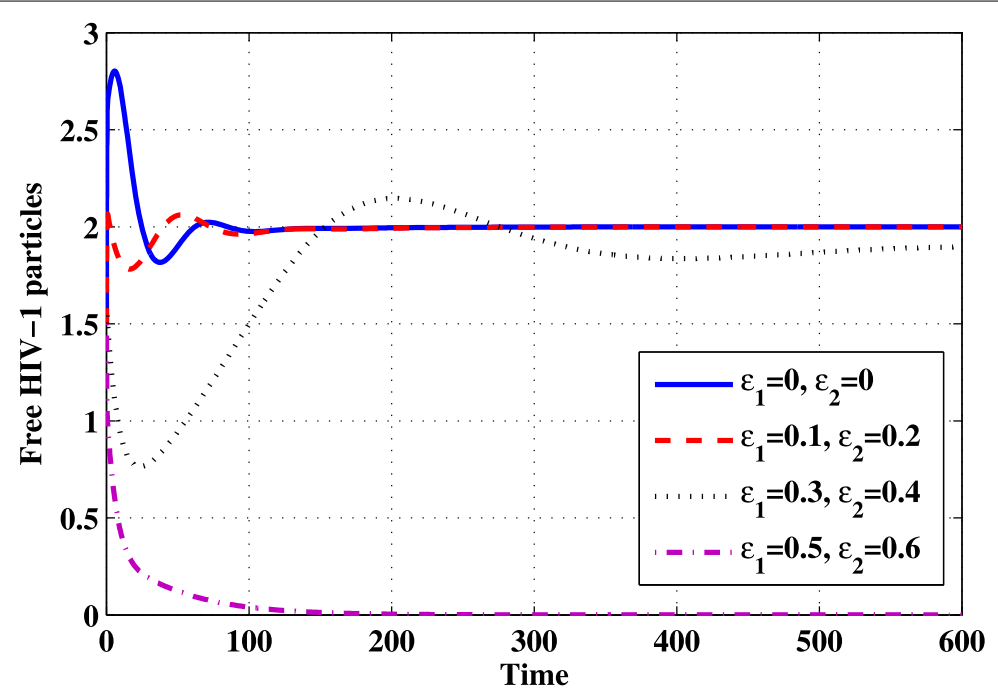

Figure 11 The concentration of free virus particles for system (66)-(71)

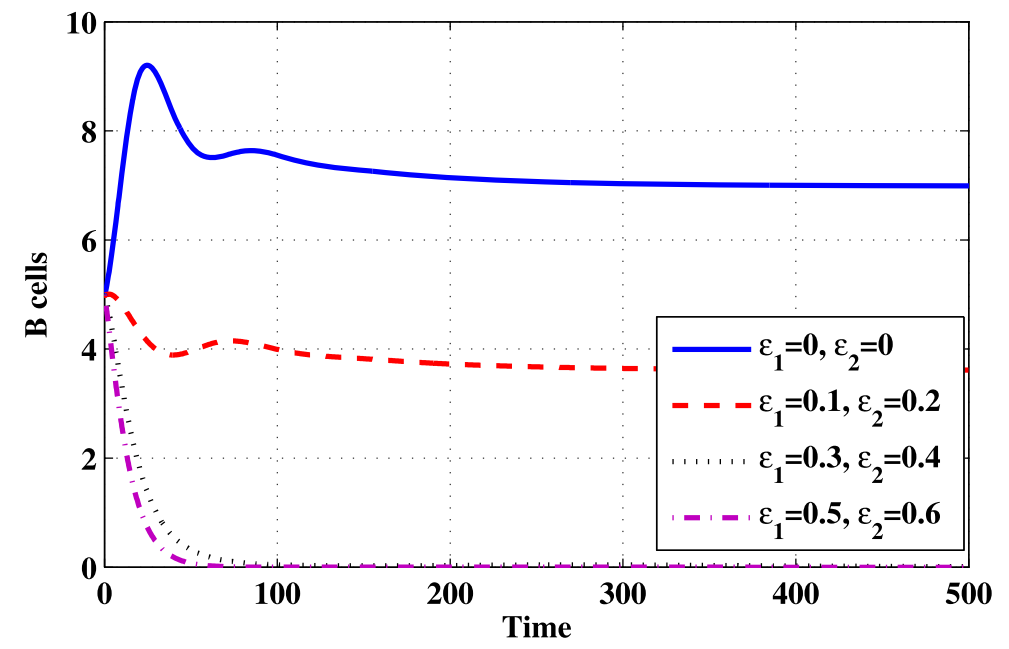

Figure 12 The concentration of B cells for system (66)-(71)

Table 2 The values of steady states $R_{0}$ and $R_{1}$ for model (66)-(71) with different values of $\varepsilon_{1}$ and $\varepsilon_{2}$

\begin{tabular}{llll}
\hline Drug & Steady states & $R_{0}$ & $R_{1}$ \\
\hline$\varepsilon_{1}=0, \varepsilon_{2}=0$ & $\Pi_{2}=(734.586,4.09194,4.77393,29.0396,2.0,7.01238)$ & 3.01635 & 2.16484 \\
$\varepsilon_{1}=0.1, \varepsilon_{2}=0.2$ & $\Pi_{2}=(734.586,3.68275,4.29654,26.1356,2.0,3.36892)$ & 2.17177 & 1.55868 \\
$\varepsilon_{1}=0.3, \varepsilon_{2}=0.4$ & $\Pi_{1}=(801.457,2.14802,2.50603,15.244,1.36614,0)$ & 1.26687 & 0.909233 \\
$\varepsilon_{1}=0.5, \varepsilon_{2}=0.6$ & $\Pi_{0}=(1001.98,0,0,0,0,0)$ & 0.60327 & 0.432968 \\
\hline
\end{tabular}

Using the data in Table 1 , we have $\varepsilon_{e}^{\text {crit }}=0.668473$.

-Effect of the time delay on the stability of the system

Choosing $\varepsilon_{1}=\varepsilon_{2}=0, \lambda_{i}=0.000625$, and $r=0.05$. The initial conditions are considered to be $(s(0), w(0), y(0), u(0), p(0), x(0))=(850,2,3,17,1,5)$. Figures $13-18$ and Table 3 show 


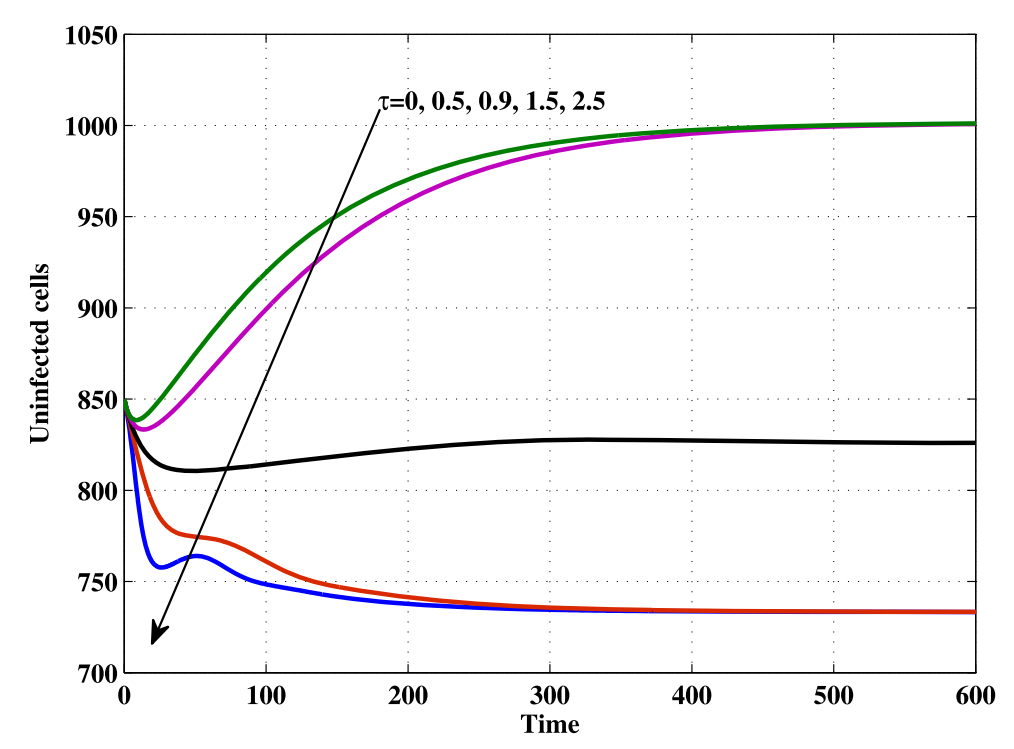

Figure 13 The concentration of uninfected CD4+ $\mathrm{T}$ cells for system (66)-(71)

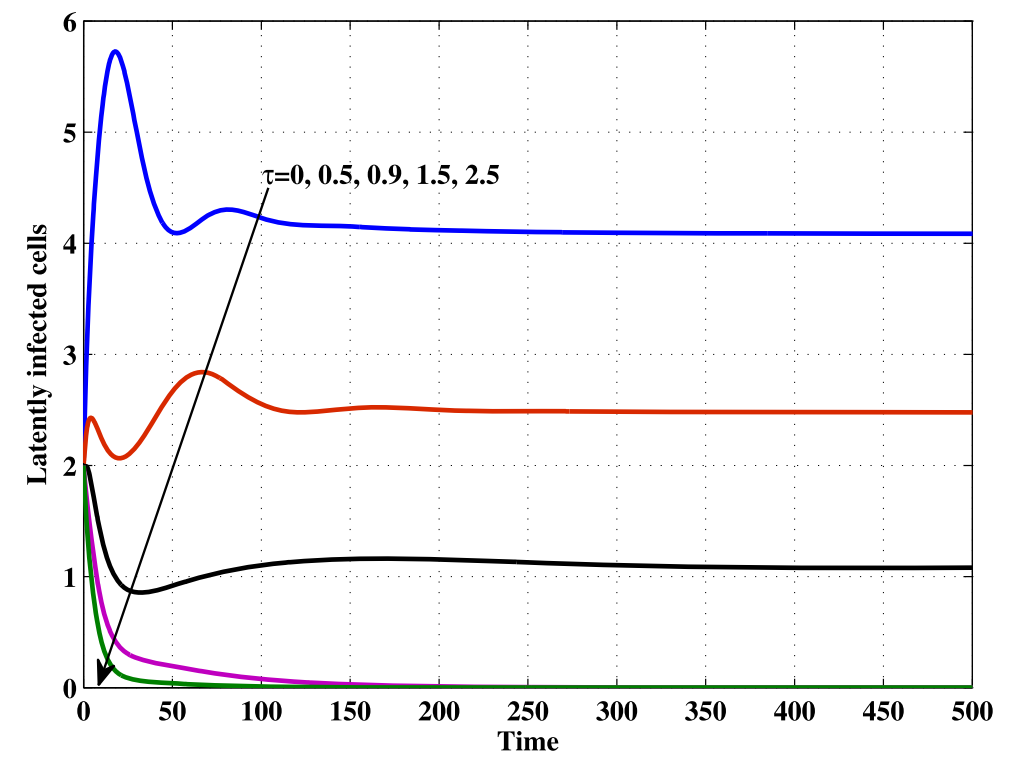

Figure 14 The concentration of latently infected cells for system (66)-(71)

the effect of the time delay parameter $\tau$ on the stability of $\Pi_{0}, \Pi_{1}$, and $\Pi_{2}$. Clearly, the parameter $\tau$ has similar effect as the drug efficacy parameters $\varepsilon_{1}$ and $\varepsilon_{2}$.

\section{Conclusion}

In this paper, we have proposed and analyzed two general nonlinear HIV infection models with humoral immune response. We have considered three types of infected cells: latently infected cells, long-lived productively infected cells, and short-lived productively infected cells. We have incorporated three discrete or distributed time delays into the models. We have considered more general nonlinear functions for the HIV-target incidence rate, pro- 


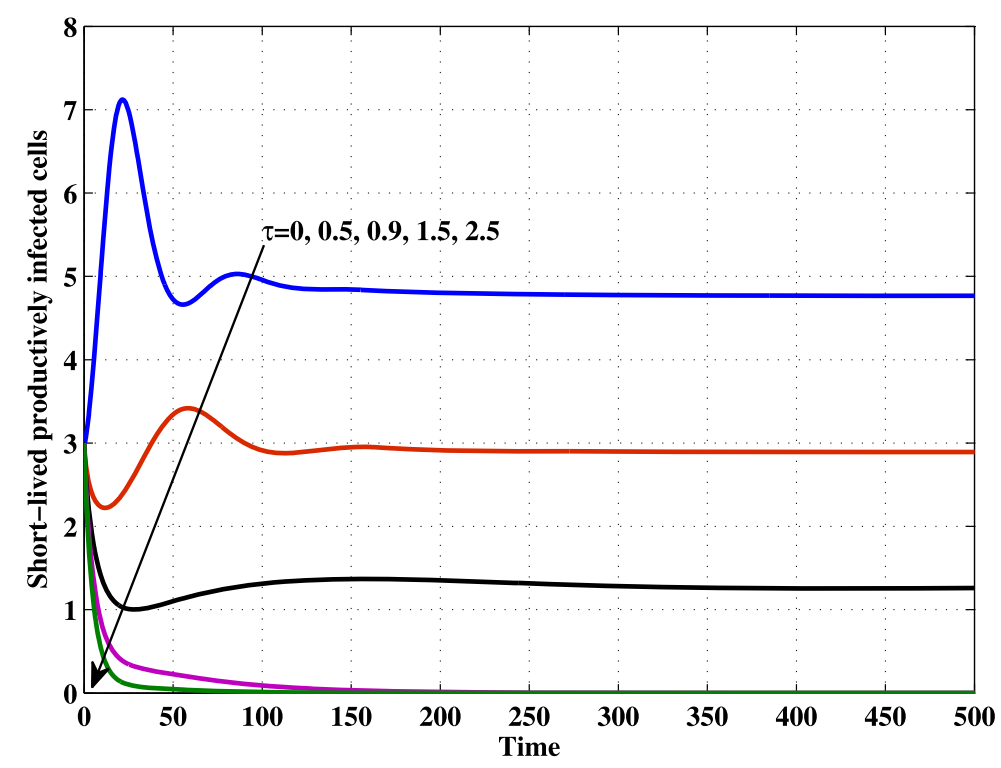

Figure 15 The concentration of short-lived productively infected cells for system (66)-(71)

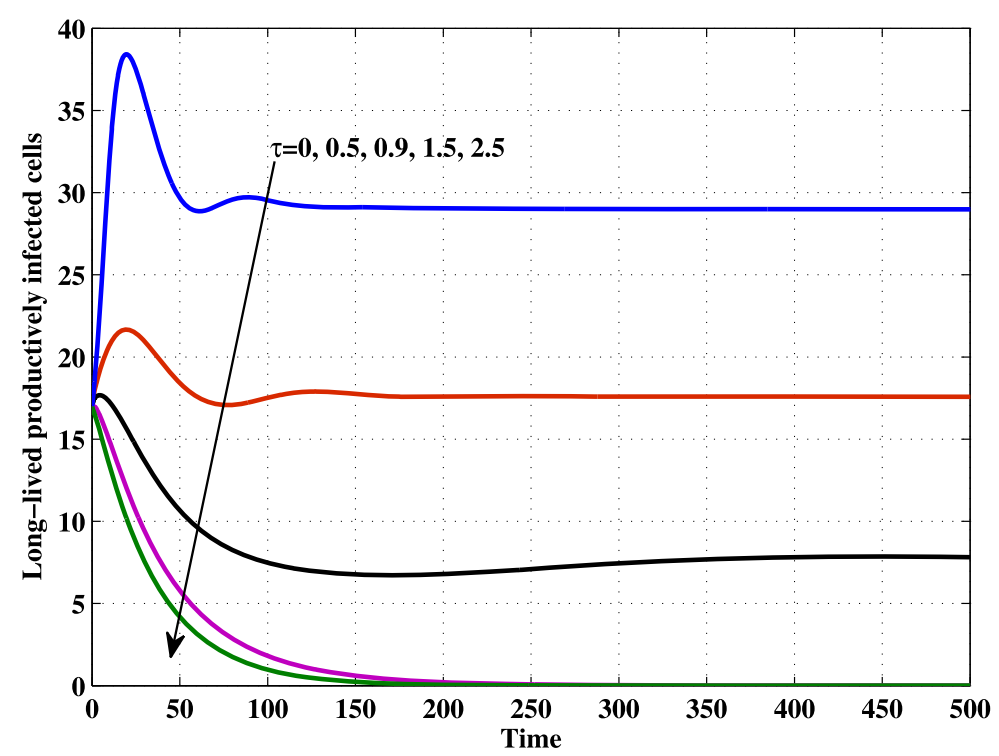

Figure 16 The concentration of long-lived productively infected cells for system (66)-(71)

duction/proliferation, and removal rates of the cells and HIV. We have derived a set of conditions on these general functions and have determined two threshold parameters: the basic reproduction number $R_{0}$ and the humoral immune response activation number $R_{1}$. We have proved the nonnegativity and ultimate boundedness of the model's solutions and the existence and stability of the model's steady states. Using Lyapunov functionals, we have established the global stability of the three steady states of the models. We have presented an example and performed some numerical simulations to support our theoretical results. 


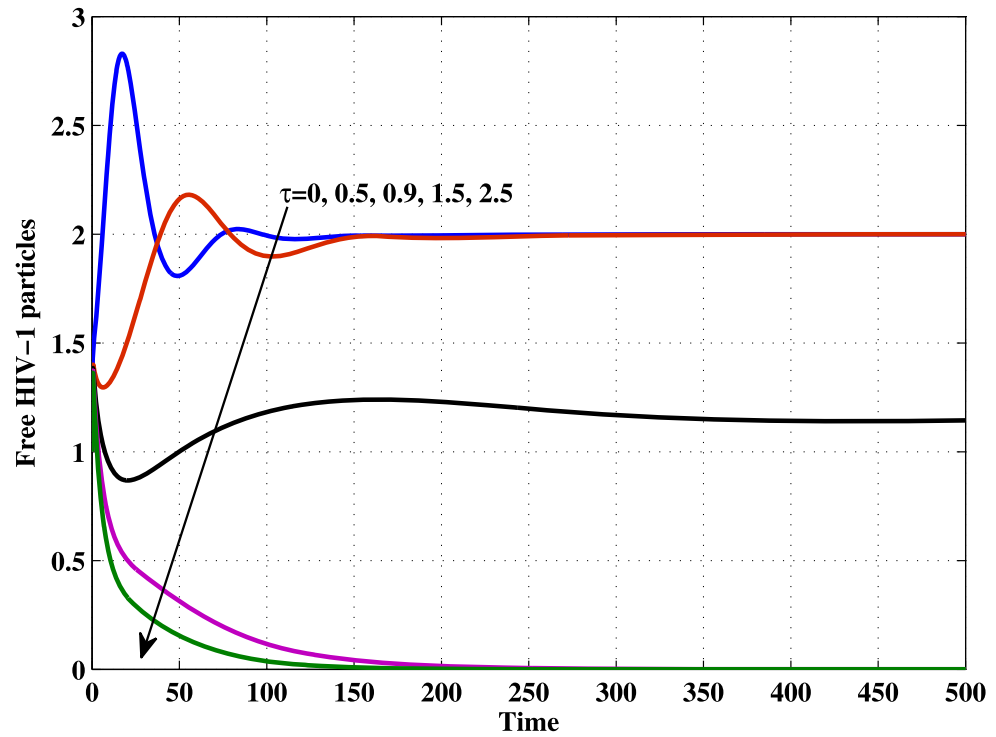

Figure 17 The concentration of free virus particles for system (66)-(71)

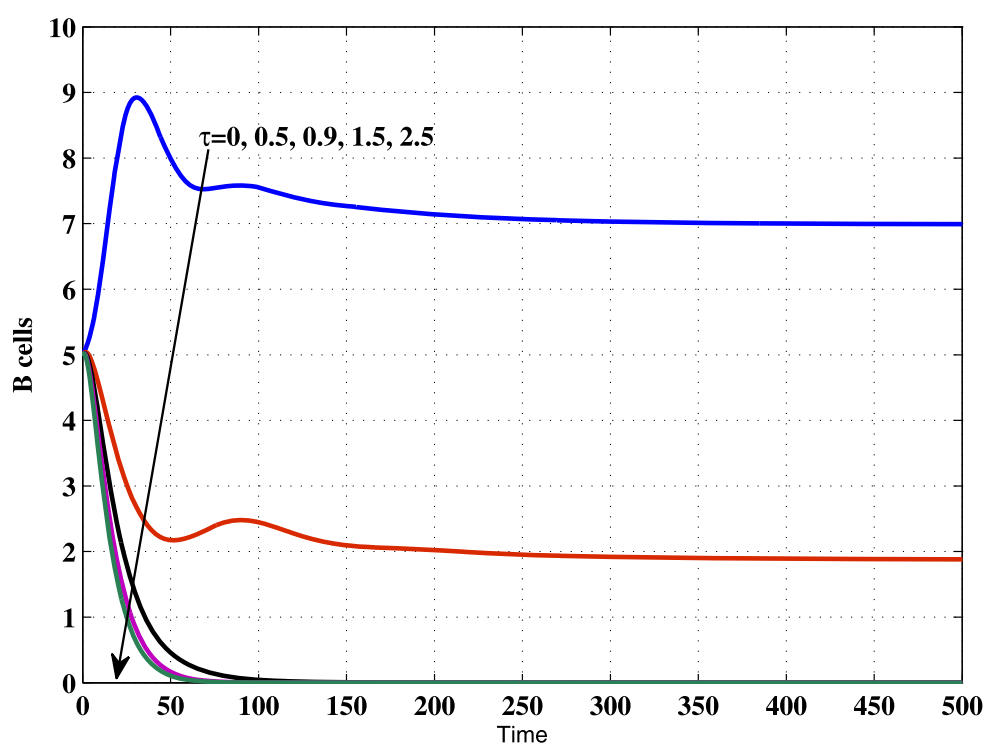

Figure 18 The concentration of B cells for system (66)-(71)

Table 3 The values of steady states $R_{0}$ and $R_{1}$ for model (66)-(71) with different values of $\tau$

\begin{tabular}{llll}
\hline Drug & Steady states & $R_{0}$ & $R_{1}$ \\
\hline$\tau=0$ & $\Pi_{2}=(734.586,4.09194,4.77393,29.0396,2.0,7.01238)$ & 3.01635 & 2.16484 \\
$\tau=0.5$ & $\Pi_{2}=(734.586,2.48189,2.89554,17.6134,2.0,1.89241)$ & 1.82951 & 1.31304 \\
$\tau=0.9$ & $\Pi_{1}=(826.24,1.09341,1.27564,7.75968,1.15901,0)$ & 1.22636 & 0.880158 \\
$\tau=1.5$ & $\Pi_{0}=(1001.98,0,0,0,0,0)$ & 0.673038 & 0.483041 \\
$\tau=2.5$ & $\Pi_{0}=(1001.98,0,0,0,0,0)$ & 0.247597 & 0.177701 \\
\hline
\end{tabular}

\section{Acknowledgements}

This article was funded by the Deanship of Scientific Research (DSR) at King Abdulaziz University, Jeddah. The authors, therefore, acknowledge with thanks DSR for technical and financial support. 


\section{Competing interests}

The authors declare that they have no competing interests.

\section{Authors' contributions}

All authors contributed equally to the writing of this paper. All authors read and approved the final manuscript.

\section{Author details}

${ }^{1}$ Department of Mathematics, Faculty of Science, King Abdulaziz University, Jeddah, Saudi Arabia. ${ }^{2}$ Department of Mathematics, Faculty of Science, Sohag University, Sohag, Egypt. ${ }^{3}$ Department of Mathematics, Faculty of Science, King Khalid University, Abha, Saudi Arabia.

\section{Publisher's Note}

Springer Nature remains neutral with regard to jurisdictional claims in published maps and institutional affiliations.

Received: 13 December 2017 Accepted: 13 February 2018 Published online: 09 March 2018

\section{References}

1. Wodarz, D., Nowak, M.A.: Mathematical models of HIV pathogenesis and treatment. BioEssays 24, 1178-1187 (2002)

2. Rong, L., Perelson, A.S.: Modeling HIV persistence, the latent reservoir, and viral blips. J. Theor. Biol. 260, 308-331 (2009)

3. Nowak, M.A., Bangham, C.R.M.: Population dynamics of immune responses to persistent viruses. Science $272,74-79$ (1996)

4. Nowak, M.A., May, R.M.: Virus Dynamics: Mathematical Principles of Immunology and Virology. Oxford University Press, Oxford (2000)

5. Hattaf, K., Yousfi, N.: A generalized virus dynamics model with cell-to-cell transmission and cure rate. Adv. Differ. Equ. 2016, $174(2016)$

6. Wang, J., Teng, Z., Miao, H.: Global dynamics for discrete-time analog of viral infection model with nonlinear incidence and CTL immune response. Adv. Differ. Equ. 2016, 143 (2016)

7. Kang, C., Miao, H., Chen, X., Xu, J., Huang, D.: Global stability of a diffusive and delayed virus dynamics model with Crowley-Martin incidence function and CTL immune response. Adv. Differ. Equ. 2017, 324 (2017)

8. Elaiw, A.M., Raezaha, A.A., Shehata, A.M.: Stability of general virus dynamics models with both cellular and viral infections. J. Nonlinear Sci. Appl. 10, 1538-1560 (2017)

9. Li, X., Fu, S.: Global stability of a virus dynamics model with intracellular delay and CTL immune response. Math. Methods Appl. Sci. 38, 420-430 (2015)

10. Perelson, A.S., Nelson, P.W.: Mathematical analysis of HIV-1 dynamics in vivo. SIAM Rev. 41, 3-44 (1999)

11. Roy, P.K., Chatterjee, A.N., Greenhalgh, D., Khan, Q.J.A.: Long term dynamics in a mathematical model of HIV-1 infection with delay in different variants of the basic drug therapy model. Nonlinear Anal., Real World Appl. 14, $1621-1633(2013)$

12. Yang, Y., Xu, Y.: Global stability of a diffusive and delayed virus dynamics model with Beddington-DeAngelis incidence function and CTL immune response. Comput. Math. Appl. 71(4), 922-930 (2016)

13. Huang, Y., Rosenkranz, S.L., Wu, H.: Modeling HIV dynamic and antiviral response with consideration of time-varying drug exposures, adherence and phenotypic sensitivity. Math. Biosci. 184(2), 165-186 (2003)

14. LV, C., Huang, L., Yuan, Z.: Global stability for an HIV-1 infection model with Beddington-DeAngelis incidence rate and CTL immune response. Commun. Nonlinear Sci. Numer. Simul. 19, 121-127 (2014)

15. Shu, H., Wang, L., Watmough, J.: Global stability of a nonlinear viral infection model with infinitely distributed intracellular delays and CTL immune responses. SIAM J. Appl. Math. 73(3), 1280-1302 (2013)

16. Hattaf, K., Yousfi, N., Tridane, A.: Stability analysis of a virus dynamics model with general incidence rate and two delays. Appl. Math. Comput. 221, 514-521 (2013)

17. Hattaf, K., Yousfi, N., Tridane, A.: Mathematical analysis of a virus dynamics model with general incidence rate and cure rate. Nonlinear Anal., Real World Appl. 13(4), 1866-1872 (2012)

18. Tian, X., Xu, R.: Global stability and Hopf bifurcation of an HIV-1 infection model with saturation incidence and delayed CTL immune response. Appl. Math. Comput. 237, 146-154 (2014)

19. Huang, G., Takeuchi, Y., Ma, W.: Lyapunov functionals for delay differential equations model of viral infections. SIAM J. Appl. Math. 70(7), 2693-2708 (2010)

20. Elaiw, A.M., AzOz, S.A.: Global properties of a class of HIV infection models with Beddington-DeAngelis functional response. Math. Methods Appl. Sci. 36, 383-394 (2013)

21. Elaiw, A.M.: Global properties of a class of HIV models. Nonlinear Anal., Real World Appl. 11, 2253-2263 (2010)

22. Huang, D., Zhang, X., Guo, Y., Wang, H.: Analysis of an HIV infection model with treatments and delayed immune response. Appl. Math. Model. 40(4), 3081-3089 (2016)

23. Monica, C., Pitchaimani, M.: Analysis of stability and Hopf bifurcation for HIV-1 dynamics with PI and three intracellular delays. Nonlinear Anal., Real World Appl. 27, 55-69 (2016)

24. Li, M.Y., Wang, L.: Backward bifurcation in a mathematical model for HIV infection in vivo with anti-retroviral treatment. Nonlinear Anal., Real World Appl. 17, 147-160 (2014)

25. Liu, S., Wang, L.: Global stability of an HIV-1 model with distributed intracellular delays and a combination therapy. Math. Biosci. Eng. 7(3), 675-685 (2010)

26. Elaiw, A.M., AlShamrani, N.H.: Stability of a general delay-distributed virus dynamics model with multi-staged infected progression and immune response. Math. Methods Appl. Sci. 40(3), 699-719 (2017)

27. Elaiw, A.M., Althiabi, A.M., Alghamdi, M.A., Bellomo, N.: Dynamical behavior of a general HIV-1 infection model with HAART and cellular reservoirs. J. Comput. Anal. Appl. 24(4), 728-743 (2018)

28. Elaiw, A.M., Almuallem, N.A.: Global dynamics of delay-distributed HIV infection models with differential drug efficacy in cocirculating target cells. Math. Methods Appl. Sci. 39, 4-31 (2016) 
29. Alshorman, A., Wang, X., Meyer, J., Rong, L.: Analysis of HIV models with two time delays. J. Biol. Dyn. 11(S1), 40-64 (2017)

30. Wang, X., Tang, S., Song, X., Rong, L.: Mathematical analysis of an HIV latent infection model including both virus-to-cell infection and cell-to-cell transmission. J. Biol. Dyn. 11(S2), 455-483 (2017)

31. Wang, X., Elaiw, A.M., Song, X.: Global properties of a delayed HIV infection model with CTL immune response. Appl. Math. Comput. 218, 9405-9414 (2012)

32. Callaway, D.S., Perelson, A.S.: HIV-1 infection and low steady state viral loads. Bull. Math. Biol. 64, 29-64 (2002)

33. Buonomo, B., Vargas-De-Le, C.: Global stability for an HIV-1 infection model including an eclipse stage of infected cells. J. Math. Anal. Appl. 385, 709-720 (2012)

34. Wang, H., Xu, R., Wang, Z., Chen, H.: Global dynamics of a class of HIV-1 infection models with latently infected cells. Nonlinear Anal., Model. Control 20(1), 21-37 (2012)

35. Korobeinikov, A.: Global properties of basic virus dynamics models. Bull. Math. Biol. 66, 879-883 (2004)

36. Pankavich, S.: The effects of latent infection on the dynamics of HIV. Differ. Equ. Dyn. Syst. (2015). https://doi.org/10.1007/s12591-014-0234-6

37. Elaiw, A.M., AlShamrani, N.H.: Global stability of humoral immunity virus dynamics models with nonlinear infection rate and removal. Nonlinear Anal., Real World Appl. 26, 161-190 (2015)

38. Hlavacek, W.S., Stilianakis, N.I., Perelson, A.S.: Influence of follicular dendritic cells on HIV dynamics. Philos. Trans. R. Soc. Lond. B, Biol. Sci. 355, 1051-1058 (2000)

39. Hale, J.K., Lunel, S.M.V.: Introduction to Functional Differential Equations. Springer, New York (1993)

40. Yang, X., Chen, L.S., Chen, J.F.: Permanence and positive periodic solution for the single-species nonautonomous delay diffusive models. Comput. Math. Appl. 32(4), 109-116 (1996)

\section{Submit your manuscript to a SpringerOpen ${ }^{\circ}$ journal and benefit from:}

- Convenient online submission

- Rigorous peer review

- Open access: articles freely available online

- High visibility within the field

- Retaining the copyright to your article

Submit your next manuscript at $\gg$ springeropen.com 